Western Kentucky University TopSCHOLAR ${ }^{\circledast}$

Fall 2015

\title{
The Examination of Enrichment Using Space and Food for African Elephants (Loxodonta Africana) at the San Diego Zoo Safari Park
}

Charlotte Hacker

charlotte.hacker230@topper.wku.edu

Follow this and additional works at: http://digitalcommons.wku.edu/theses

Part of the Biology Commons, and the Zoology Commons

\section{Recommended Citation}

Hacker, Charlotte, "The Examination of Enrichment Using Space and Food for African Elephants (Loxodonta Africana) at the San Diego Zoo Safari Park" (2015). Masters Theses \& Specialist Projects. Paper 1547.

http://digitalcommons.wku.edu/theses/1547 


\author{
A Thesis \\ Presented to \\ The Faculty of the Department of Biology \\ Western Kentucky University \\ Bowling Green, KY
}

In Partial Fulfillment

Of the Requirements for the Degree

Master of Science

By

Charlotte Evelyn Hacker

December 2015 
EXAMINATION OF ENRICHMENT USING SPACE AND FOOD FOR AFRICAN ELEPHANTS (LOXODONTA AFRICANA) AT THE SAN DIEGO ZOO SAFARI PARK

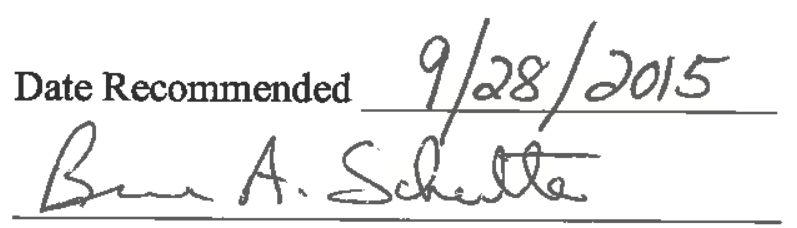

Bruce A. Schulte, Director of Thesis
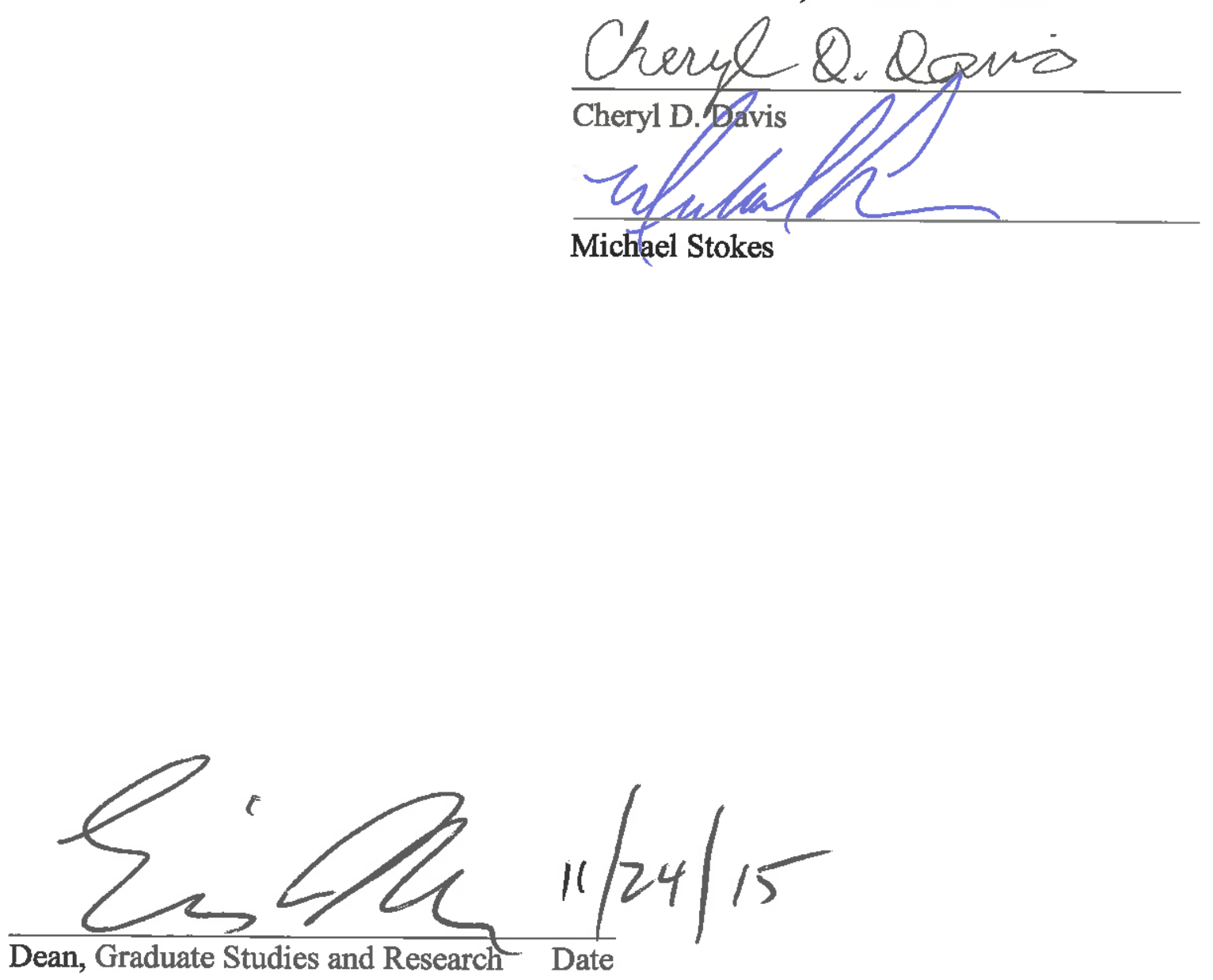


\section{ACKNOWLEDGEMENTS}

This thesis could not have been completed without the aid of so many individuals, both directly and indirectly related to this study. First and foremost, I would like to thank my advisor, Dr. Bruce Schulte, not only for his guidance, but for taking a chance on me in the first place. I would also like to heavily thank Dr. Lance Miller, who made this study a reality and has provided invaluable feedback to me as a researcher. My committee members, Dr. Cheryl Davis and Dr. Mike Stokes also deserve my utmost appreciation for their contribution to this work.

Thank you to the Heller family for selflessly funding my position with San Diego Zoo Global, and to WKU's Graduate School and the Department of Biology for research funds. To the elephant management staff at the San Diego Zoo Safari Park, I cannot put into words how much your support was appreciated. Thank you to the staff at the Institute of Conservation Research, who welcomed me and provided me with many of the most interesting lunch conversations I will ever have the pleasure of being a part of.

I additionally would like to thank my parents and grandmother, who did not even blink when their little girl from Delaware wanted to spend months away from home in Africa because she "just really liked elephants." I doubt I would have ever found my passion without your assistance and bravery. An additional shout-out is rightfully due to Steven Zielen. Your support in my endeavors has led to more than I ever could have dreamed. I would also like to thank the many friends in both California and Kentucky who have become like family and have provided endless encouragement, love, and laughs. 


\section{TABLE OF CONTENTS}

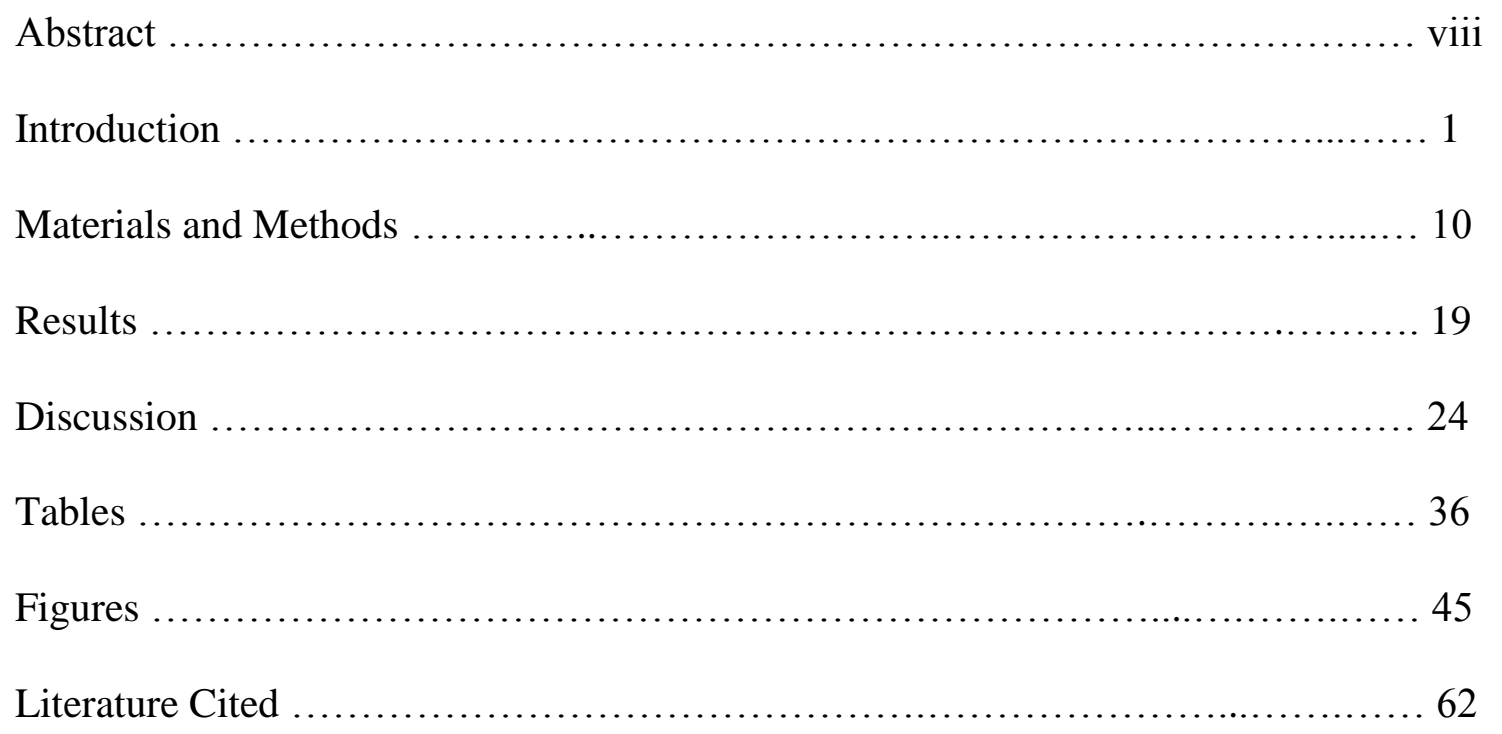




\section{LIST OF FIGURES}

Fig 1. A Google Earth image of the elephant exhibit at the San Diego Zoo Safari Park

Fig 2. The GPS anklets used to assess elephant walking distances $\ldots \ldots \ldots \ldots \ldots \ldots \ldots \ldots 46$

Fig 3. The exhibit treatments used to assess space and food effects ................. 47

Fig 4. Images of the training of younger elephants to accept GPS anklets ............ 48

Fig 5. Images of the testing of the GPS anklet design ........................... 49

Fig 6. The average total distance walked for each treatment .................................50

Fig 7. The average Shannon-Wiener Index for each treatment ...................... 51

Fig 8. The relationship between average Shannon-Wiener Index and total average

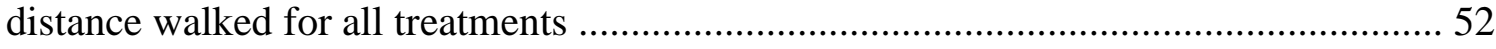

Fig 9. The relationship between average Shannon-Wiener Index and total average

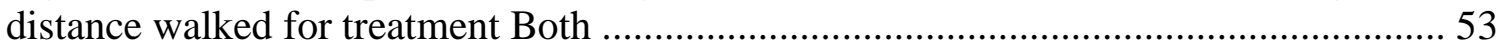

Fig 10. The relationship between average behavioral richness and average total distance

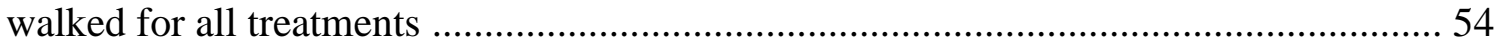

Fig 11. The relationship between average behavioral evenness and average total distance walked for all treatments 55

Fig 12. The relationship between total average distance walked and dominance rank across treatments

Fig 13. The relationship between average Shannon-Wiener Index and dominance rank across treatments

Fig 14. The relationship between average behavioral richness and dominance rank across treatments 58

Fig 15. Sociograms representative of the associations between elephant individuals in

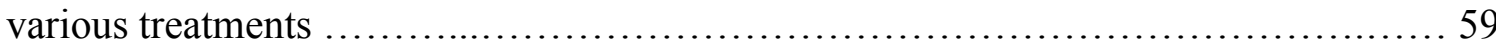

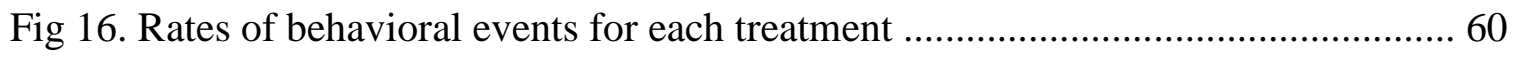

Fig 17. Effects of tossed produce and pellets on walking distances ........................... 61 


\section{LIST OF TABLES}

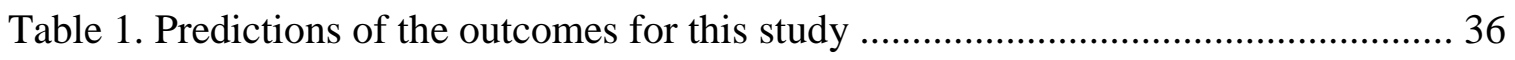

Table 2. Information regarding the elephants at the San Diego Zoo Safari Park.......... 37

Table 3. The data sheet used to record behaviors for this study ........................ 39

Table 4 . The ethogram of states and events used for this study $\ldots \ldots \ldots \ldots \ldots \ldots \ldots \ldots . \ldots \ldots$

Table 5. The p-values associated with differences in various behavioral event categories

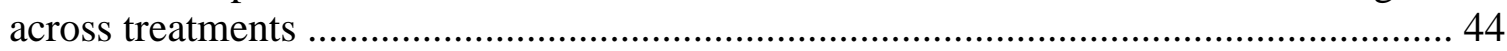




\section{EXAMINATION OF ENRICHMENT USING SPACE AND FOOD FOR AFRICAN ELEPHANTS (LOXODONTA AFRICANA) AT THE SAN DIEGO ZOO SAFARI PARK}

Charlotte Hacker

December 2015

68 Pages

Directed by: Dr. Bruce A. Schulte, Dr. Michael Stokes, and Dr. Cheryl Davis

Department of Biology Western Kentucky University

Concern for elephant welfare in zoological facilities has prompted a number of exhibit and management modifications, including those involving enrichment.

Knowledge of how these changes impact measures of health and wellbeing, such as elephant movement and behavior, is crucial as the effects of multiple enrichment types and their interactions are largely understudied. The present study used observations and GPS unit collected data to determine the effects of space and food on the walking distance and behavior of thirteen African elephants, whose dominance structure was ascertained by the handlers at the San Diego Zoo Safari Park (SDZSP). This facility has two exhibits of approximately equal size. Three treatments were created to assess the effects of food and space enrichment: (1) access to half of the exhibit with food (Half); (2) access to both yards with food in one yard, or half the total exhibit space (Both/Half); and (3) access to both yards with food in both (Both). To account for mirrored effects, the reverse for Half and Both/Half were also completed. Significant differences across treatments were revealed for average total walking distances, which varied among elephants belonging to different dominance groups. Overall, treatment Both evoked the most diverse behavior. Walking and behavioral data were related, as were walking distances and elephant dominance rank. No such relationships were found between dominance and behavioral measures. The information obtained from this study has direct 
implications for the management of the SDZSP elephants and could be applicable for elephants at other facilities that consider the choices between increasing the size of exhibits and the use of other forms of enrichment. 


\section{INTRODUCTION}

The African elephant (Loxodonta africana) made its debut in North America about 200 years ago and has since become a common flagship species in zoos around the United States (Olson, 2004). The Association of Zoos and Aquariums (AZA) has instituted a number of mandated policies in the area of elephant management and care to make certain that elephants receive adequate treatment and attention. Member institutions of the AZA are required to fulfill numerous components to ensure the proper welfare of animals including adequately trained staff, annual completion of behavioral profiles, and approved emergency response protocols (Olson, 2004). A further consideration involves the physical and psychological wellbeing of the elephants at these facilities (Maple, 2014; Tarou and Bashaw, 2007).

Zoological institutions housing elephants are in general agreement that improvements in elephant management are necessary; however, there is little consensus on what improvements are needed (Hutchins, 2006). Substantial changes in the size and complexity of exhibits have taken place in recent decades (Leighty et al., 2010) in addition to the building of new exhibits all together. Examples of facilities with new exhibits for elephants include the San Diego Zoo in 2009 (Schwartz, 2009), the Los Angeles Zoo (Lewis, 2010) and Dallas Zoo in 2010 (Dallas Zoo, 2013), the Cleveland Metroparks Zoo in 2011 (Cleveland Metroparks Zoo, 2013), the Denver Zoo in 2012 (Denver Zoo, 2013) and Cheyenne Mountain Zoo in 2013 (Cheyenne Mountain Zoological Society, 2013). Exhibits currently under construction can be found at the Oregon Zoo (Oregon Zoo, 2014) and at the Audubon Zoo (Audubon Nature Institute, 2013), both due to open in 2015. More recently, a multi-institutional study among 72 North American zoos entitled the Elephant Welfare Project has collected various data 
measurements and information regarding Asian and African elephants in an effort to improve their overall welfare. The project was the largest zoo animal welfare study to date and was funded by the Institute of Museum and Library Services (IMLS). One component of this study specifically looked at walking distances and recumbence behavior (Holdgate, 2015). A plethora of information pertaining to elephant welfare will emanate from the IMLS project. Still, detailed examinations of individual zoos and their elephants are vital in making specific management assessments and suggestions, and analysis of differences directly before and after enrichment changes is lacking.

In recent decades, zoos have been faced with mounting criticism regarding the welfare of their elephants (Clubb et al., 2008; Clubb and Mason, 2003b; Cohn, 1992; Veasey, 2006). Such concerns have prompted research into environmental enrichment (Wells, 2009). According to the AZA, enrichment is defined as "a dynamic process for enhancing animal environments within the context of the animals' behavioral biology and natural history" (Colbert, 2010). There are five categories of enrichment: social, feeding, physical, occupational, and sensory (Tarou and Bashaw, 2007). The goal of environmental enrichment is to increase behavioral choices for animals on exhibit and to encourage species-appropriate behaviors, ultimately enhancing their welfare. Care must be taken in the creation of an enrichment program as it can be costly, deplete resources, and/or not have a lasting benefit (Cipreste et al., 2010). To be successful over the long term, programs must also be variable and unpredictable to the animals of concern (Cipreste et al., 2010). This is particularly important for elephants, as they are cognitively advanced (Bates, 2008) and have exhibited associative learning, (Desmond and Laule, 1991), insight learning (Foerder et al., 2011), cooperative learning (Plotnik et al., 2011), 
and habituation (Goodyear, 2015). Past enrichment studies with elephant subjects have examined the effects of housing (Posta et al., 2013), feeder balls (Rees, 2009), water spray devices (Mellen et al., 1981), and feeding (Bjork, 2011; Posta et al., 2013; Stoinski et al., 2000) on behavioral responses including overall activity budgets, animal visibility to zoo guests, and stereotypic behavior (i.e., stereotypies, see Mason, 1991).

In the wild, animals are regularly exposed to a dynamic and fluctuating array of stimuli (Wells, 2009). In zoos, animals are generally exhibited in a predictable environment, which can lead to atypical behaviors (Laule, 2003, RSPCA, 2007). The addition of stimuli into an exhibit area can effectively promote enrichment and create long-term benefits, thereby enhancing the welfare of the animals (Laule, 2003). Enrichment has been shown to have positive effects on Asian elephants (Elephas maximus) (Tresz, 2006). The Phoenix Zoo in Arizona modified its behavioral management program and added enrichment to its Asian elephant exhibit, including extra holding pens, more time outside, a new wallow, scratching post, and additional sand. As a result, the elephants' foraging times increased, foot problems and aggression decreased, and a greater variety of natural behaviors were performed. Enrichment has also been shown to have positive results on American black (Ursus americanus) and brown (Ursus arctos) bears (Carlstead et al., 1991). The introduction of manipulative objects in their exhibit increased appetitive behavior and decreased stereotypic behavior. Enrichment caused an increase in locomotion in Hanuman langur monkeys (Presbytis entellus) (Little and Sommer, 2002) and stress hormones were found to be lower in both rats (Rattus) (Belz et al., 2003) and small cats (tigrina (Leopardus tigrinus) and margay (Leopardus wiedii)) (Moreira et al., 2007). However, some studies indicate less positive effects. For 
example, enrichment was found to have no effect on American minks (Neovison vison) (Dallaire et al., 2011) and greater unpredictability in daily routine increased stereotypic behaviors in a female Asian elephant (Elzanowski and Sergiel, 2006). Still, mostly positive results have occurred with the use of enrichment, and additional information is needed on the interaction between exhibit space, which provides more room to move, and complexity, which can provide more behavioral opportunities.

Relatively smaller exhibit sizes negatively impact behavior in a variety of animals such as non-human primates (Hosey, 2005), Asian elephants (Koene, 1995), chickens (Gallus gallus domesticus), and horses (Equus ferus caballus) (Levy, 1944). Stress hormones were found to be elevated in sheep (Ovis aries) (Horton et al., 1991) and primates (Hediger, 1955) in smaller spaces. Less locomotion was noted in pigs (Sus scrofa) (Pearce and Patterson, 1993) and stereotypic pacing was observed in large cats (Clubb and Mason, 2003b).

Abiotic factors, such as season, weather, and time of day, also can alter the behavior of animals in captivity. Elephant behavior and physiology can be affected by the time of day, season, and housing conditions (Posta, 2011; Schulte, 2000). For example, a number of self-directed behaviors were found to be more prevalent during sun exposure than no-sun exposure (Posta, 2011). These behaviors included dusting, digging, and rubbing on objects.

Biotic factors other than food enrichment also can influence behavior, such as social group composition, dominance hierarchies, and health. African elephant social groups follow a fission-fusion society (Archie et al., 2006a). Female African elephants live in matrilineal herds led by a matriarch. The matriarch is generally the oldest female 
of the group, and her knowledge is communicated to her group members (McComb et al., 2001). Female elephants and their level of kinship determine the social importance of one individual to another, thus driving predictable trends in the fission and fusion of social groups in the wild (Archie et al., 2006b). Male African elephants leave their natal family during adolescence and form male groups (Evans and Harris, 2008). The group in which an adolescent or adult male associates is dependent upon his age, size, and sexual state. Heightened sexual and aggressive activity occurs during a period known as musth. During this time, males will move away from their group in search of estrous females (Poole, 1996). Elephants within zoological institutions are generally housed in a greater range of group sizes, age structures, and degrees of relatedness than they would in a wild environment (Schulte, 2000). Elephants may interact with each other differently depending on their level of relatedness, and the rate of social interaction compared to density. Thus, the influence of elephants on one another is an important consideration when manipulating zoological environments, particularly because social relations in elephants are cryptic and difficult to assess (Wittemyer et al., 2005). Social relationships can be determined via behavioral observations (Altmann, 1974; Archie et al., 2006a; Gadgil and Nair, 1984; Pepper et al., 1999; Wittemyer and Getz, 2007; Wittemyer et al., 2007), GPS technology (Hacker et al., 2015), and through keeper knowledge (Freeman et al., 2010; Hacker et al., 2015). Input from animal care staff is vital in zoological settings as keepers can provide invaluable information of the animals in their care and thus aid in management decisions (Hosey, 2008).

The foraging behavior of elephants is influenced by gender, environment, and body size (Shannon et al., 2006). When it comes to diet, larger herbivores are more likely 
to seek lower quality forage in larger amounts than higher quality food in smaller amounts (Senft et al., 1987; Shannon et al., 2006). This is because the benefits received from the abundance of food outweigh the energy and costs related to searching for higher quality food. Adult female elephants are generally more selective about their food than adult male elephants in that females seek out higher quality food sources to aid in reproduction (Shannon et al., 2006; Stokke and Du Toit, 2002). During the dry season, bull elephants use a greater diversity of habitats and stray farther from water sources. However, this is not likely to be because of intraspecific competition over food, but rather a way for bull elephants to avoid others in musth to diminish the possibility of conflict and to access feeding locations, which have more high quality, accessible, and nutritional food (Stokke and Du Toit, 2002).

The diet of African elephants varies through seasons. During the rainy season, green grasses and herbs are generally selected. During the dry season, the intake of browse foliage and roots increases (O'Connor et al., 2007). Most food selected is that which provides rapid nutrient uptake as the retention time of food for African elephants is about 14 (Johansson, 2009) to 46 hours (Rees, 1982). A measurement related to foraging ability and food quality is body mass or weight. Weight can be used as indicator of health in elephants. An elephant who is not able to fulfill their nutritional intake over time will have a low body weight and overall poor body function and condition (Buckley, 2008).

Generally, elephants cover vast distances in their native habitat (Wittemyer et al., 2007). Elephants move to access resources such as food, water, and shelter, to avoid threats (e.g., aggressive conspecifics, predators, or humans), and to locate conspecifics. Because resources are provided to elephants in captivity, they may not move more when 
just given greater space. However, the manipulation of both space and the distribution of resources in captive settings could increase activity levels.

Movement is an important aspect to consider when managing elephants as numerous ailments such as foot issues, arthritis, and obesity are linked to a lack of exercise (Clubb and Mason, 2003a; Miller et al., 2011; Veasey, 2006). More active animals may also be more appealing to zoo visitors. For example, Margulis and colleagues (2003) found that visitor interest was higher when viewing active versus inactive felids. However, it is important that movements and activity are species appropriate and not stereotypic motions (Altman, 1998), especially because zoos provide one of the few opportunities for people to view animals up-close and inspire conservation of the natural world (Beardsworth and Bryman, 2001). Miller (2012) found that guests who saw a video of a tiger pacing were less likely to support zoos and state that the tiger had good welfare, than guests who saw a video of an inactive tiger. Environmental enrichment in the form of space and food may encourage species-appropriate movement and behavior.

The assessment of behavior has been widely used in an attempt to quantify animal welfare and the effectiveness of enrichment (Marchant-Forde, 2015; Shepherdson, 2010). The level of behavioral diversity exhibited by an animal is indicative of the behavioral opportunities available. Enrichment helps to increase behavioral opportunities and control, so behavioral diversity can measure the effectiveness of enrichment (Shepherdson, 2010). The diversity of behavior has been assessed in a number of ways including richness, repertoire size, and diversity indices. The Shannon-Wiener Index (Shannon and Weaver, 1949) has historically been used in ecological frameworks, but it 
also has been modified and used in the animal behavior field with nocturnal mammals (Clark and Melfi, 2011), small cats (Shepherdson et al., 1993), and now for elephants with this study. The Shannon-Wiener diversity index is an appropriate measure of behavioral diversity. It is calculated using both the richness, or number of different behaviors completed, and the evenness, or distribution of behaviors. In the calculation of the Shannon-Wiener index, different combinations of richness and evenness can yield very similar values. When assessing the effects of enrichment, the desired outcomes on behavior should be clear. That is, whether more behaviors are desired, or a greater balance among behaviors, or some combination thereof. In addition to these two factors, the types of behaviors also may be important, and none of the aforementioned measures or richness, repertoire size, or the $\mathrm{S}-\mathrm{W}$ index consider type.

The goal of the present study was to determine the effects of exhibit space and food on thirteen African elephants at the San Diego Zoo Safari Park (SDZSP). This study was pre-designed by elephant management staff and associated scientists belonging to the zoo's Institute of Conservation Research. To my knowledge, this is the first study to manipulate space and strategic food type and placement and then measure the responses of walking and other behaviors by elephants. The SDZSP's elephant enclosure has one exhibit consisting of two nearly identical yards of approximately equal size. Three treatments to assess the effects of food and space enrichment were deployed: access to one yard with food (Half); access to both yards with food in one yard, or half the total exhibit space (Both/Half); and access to both yards with food in both (Both). To account for mirrored effects, the reverse for Half and Both/Half were also completed (HalfMirrored and Both/Half-Mirrored). Three different types of food presentation were 
deployed during each trial - pre-set hay piles and scattered browse set out prior to elephant release into the respective exhibit space, tossed pellets and produce, and tossed hay piles. While total food amount (6 bales of hay total) was constant for each of the treatments, the distribution of food for treatment Both was more spatially dispersed than for treatments Half, Half-Mirrored and Both/Half, Both/Half-Mirrored.

Overall, the expectation was that elephants in the treatment with the greatest space and distributed food (Both) would exhibit the highest levels of walking, and the largest behavioral diversity, evenness and richness values, with behavioral richness and evenness contributing equally to the behavioral diversity value. It was anticipated that elephants would walk farther distances when provided a larger space, and that walking would increase as food became more spatially dispersed within the provided exhibit space. However, walking was also expected to be variable among elephants belonging to different dominance groups, as motivations for the movement of subordinate animals is likely impacted by those more dominant. Behavior was predicted to be variable between treatments, and between individuals, primarily because of dominance. For example, access to both yards provided an additional set of rock structures, a water hole, and various substrates. Animals would likely walk more and exhibit more behavioral activities when given access to both yards, but subordinate animals especially would be given the opportunity to use a resource that may be occupied in one yard but not in the other. Elephants in the treatment with the least space (Half) were predicted to display the lowest levels of each measure, and evenness and richness were again expected to contribute equally to behavioral diversity (Table 1). 


\section{MATERIALS AND METHODS}

\section{Study Site and Subjects}

The present study was conducted from March to July of 2014 at the San Diego Zoo Safari Park (SDZSP) in San Pasqual Valley near Escondido, California (33.099703 $\left.\mathrm{N} / 113.001525^{\circ} \mathrm{W}\right)$. Escondido lies in a valley along the coastal mountains of Southern California. It is approximately 18 miles inland and 30 miles northeast of San Diego, California (Escondido, City of Choice, 2013). According to Weather Underground, the average temperature and precipitation for the months of the study were as follows - for March, $15.6^{\circ} \mathrm{C}$ and $2.12 \mathrm{~cm}$, for April the averages were $16.8^{\circ} \mathrm{C}$ and $2.24 \mathrm{~cm}$, for May the averages were $20.6^{\circ} \mathrm{C}$ and $0 \mathrm{~cm}$, for June the averages were $19.8^{\circ} \mathrm{C}$ and $0 \mathrm{~cm}$, and the averages for July were $22.6^{\circ} \mathrm{C}$ and $0.38 \mathrm{~cm}$ (Weather Underground, 2015).

In 2003, SDZSP and the Lowry Park Zoo in Florida imported eleven African elephants at risk for culling in Swaziland due to overpopulation, habitat destruction, and threats to critically endangered species (Andrews et al., 2004). All elephants were estimated to be 12 to 13 years of age. One male and six females went to the SDZSP, one of whom was pregnant upon arrival. The male sired calves with all but one of the five females and the herd grew to 17 elephants. In 2012, the male, two females, and two calves were moved to the Reid Park Zoo in Arizona and a different male from the Lowry Park Zoo in Florida was introduced to the remaining elephants at the SDZSP. At the time of the present study, the herd at the SDZSP included thirteen African elephants consisted of four adults, one sub-adult, three juveniles, and six calves (for age determinations see Loizi et al., 2009). The elephants were managed via protected contact with a trust-based training system. The exhibit is entitled 'Elephant Valley' and consisted of a 0.26 hectare 
area that can be divided into east and west halves ( 0.14 hectares and 0.12 hectares) by an electronic remote controlled gate (Fig. 1) (San Diego Zoo Safari Park, 2013). Elephants were provided the east yard, west yard, or both yards depending upon the keepers' and elephants' needs and this varied from day to day. For example, while the keepers cleaned the west yard of the exhibit space, the elephants were kept on the east yard. The elephants would be switched to the west yard while keepers cleaned the east yard, and after cleaning the elephants may be provided with both yards, split into family groups between the two yards, or all kept in just one yard.

\section{Preliminary observations}

Early summer of 2013 was used as a training and acclimation period. This included ad libitum observations in order to assess elephant identities. Elephants can be identified by physical features such as marks on the ears, wrinkles, tusk and tail shapes, and body size (Table 2). Meetings with elephant management staff and other SDZSP employees were held frequently to solidify study protocol. In late summer of 2013, practice behavioral observations were conducted during various times of the day. The GPS anklets also were constructed at this time (Excelsior Leather [Fallbrook, California]) (Fig. 2).

Preliminary behavioral data collection continued throughout the fall and winter of 2013 into early 2014. Training regarding GPS anklet wear was added into the elephants' regular training schedule. On two separate occasions, once in October 2013 and once in November 2013, trial run-throughs were completed to assess the applicability of the study schedule. It was determined that $22 \mathrm{hr}$ was the maximum time possible for keepers 
to be uninvolved with the elephants before the elephants exhibited anxious behavior (i.e. herd circling, vocalizations, and loitering).

\section{Treatments}

Experimental data collection began in March of 2014. In total, five treatments to assess quality versus quantity took place, with two of those having mirrored designs (Fig. 3). Treatment Half provided access to only the west yard of the exhibit with food on that side. Treatment Half - Mirrored, provided access to only the east yard of the exhibit with food on that side. Treatment Both/Half, provided the elephants with access to both yards and with food in the west yard. Treatment Both/Half - Mirrored provided access to both yards with food in the east yard. Treatment Both provided access to both yards with food in both yards. During each treatment, $6.5 \mathrm{hr}$ of behavioral observations and $22 \mathrm{hr}$ of walking activity were collected. Treatments lasted for $22 \mathrm{hr}$ and started on various days of the week depending on the keepers' schedules until 3 trials of each of the treatments were completed, resulting in three separate rounds (herein referred to as $a, b$, and c). The order of treatments was selected randomly without replacement via a random number generator in Microsoft Excel. Treatments began at 11:00 AM and ceased the next day at 9:00 AM. All 13 subjects were kept as one social group except from 3:00 PM to 8:00 AM during which the adult male (bull) was kept in the opposing yard (treatment Half) or west bull yard (treatments Both, Both/Half) because of safety concerns for the other members of the herd when the elephant handlers were not present. For treatments where the herd had access to only one yard (treatment Half), the sub-adult male was also segregated into the west bull yard (with the exception of one trial of the Half treatment) also for the safety of the animals. 
Each treatment involved the same amount and varieties of hay, though types of browse occasionally varied depending on availability. Every treatment trial started with four bales of Bermuda grass hay (Cynodon dactylon) and two bales of Sudan grass hay (Sorghum $x$ drummondii) distributed into 16 separate piles throughout the available exhibit space. One cart of browse was then scattered throughout the space. Browse generally consisted of the branches and leaves of woody plants from the genus Ficus, though because of reduced availability, some of the treatments involved other types of browse such as bird-of-paradise (Stelitzia reginae) or panaceum (Poganatherum panaceum). The PI on the project was responsible for setting food items with occasional help from keepers to ensure consistency. In order to keep track of food pile placement throughout the study, pile location and type (bermuda, sudan, bermuda/sudan mix) were recorded onto a Google Earth image of the elephant exhibit. Supplemental food products were supplied at two different times during the treatment. At approximately 3:00 PM (4 $\mathrm{hr}$ into the treatment), produce such as celery (Apium graveolens), romaine lettuce (Lactuca sativa L. var. longifolia) and cucumbers (Cucumis sativus) and alfalfa pellets were thrown into the exhibit space respective of where hay piles and browse was initially placed. At approximately 8:00 AM the following morning ( $21 \mathrm{hr}$ into the treatment), one bale of Sudan hay was thrown into the exhibit where food was initially placed with the exception of one treatment of Both/Half, when Bermuda hay was used due to the unavailability of Sudan.

\section{Walking Activity}

To digitally document walking distance, an anklet with Global Positioning System technology was implemented (Leighty et al., 2010; Holdgate, 2015; Miller et al., 2011; 
Theiss et al., 2005). Although there are no studies testing the effect of GPS anklets on elephant behavior, Horback et al. (2012) examined the effects of GPS collars and found that they had no effect on elephant behavior. Because elephant collars are considerably bulkier and heavier than anklets, it was anticipated that GPS anklets would also reliably measure elephant movement without any adverse effects. In a prior study, five of the elephants were trained using positive reinforcement to wear GPS anklets (Miller et al., 2011). Using that same training process, three additional younger elephants were conditioned to wear GPS anklets to examine 22-h walking rates (Fig. 4). These eight comprised four adults, one sub-adult, two juveniles, and one calf. Anklets were put onto the elephants by 11:00 AM the day of the treatment and were removed soon after 9:00 AM the next morning. One keeper 'held' the elephant, while another maneuvered the anklet. A GPS tracking unit (Qstarz BT-Q1000X [Taipei, Taiwan]) was encased in a waterproof OtterBox ${ }^{\mathrm{TM}}$ DryBox 1000 [Fort Collins, Colorado] and was secured in a leather pouch in the middle of the anklet. Testing of anklet design was completed to ensure the absence of any visible discomfort, such as marks or sores, on the elephants (Fig. 5). The GPS units were reported by the manufacturing company to be accurate within $2.5 \mathrm{~m}$ and an earlier study found the units to be reliable except during severe weather (Miller et al., 2011), which never happened during the time period of this study. GPS location points were collected every $5 \mathrm{sec}$, and included time, coordinates, and related accuracy measures. The data were downloaded from the GPS unit to a PC computer and opened using the GPS device's accompanying utility software (QTravel V1 [Taipei, Taiwan]). Data were revised by removing any points with fewer than six satellites in view or a horizontal position accuracy (HDOP) score greater than two. 


\section{Behavior}

Data were collected from 11:00 AM to 1:30 PM, 2:30 PM to 5:00 PM, and 6:30 AM to 9:00 AM the next morning. Observations included focal follows using continuous sampling (states) and all occurrence sampling of specific behaviors (events) using an ethogram (Table 3) modified from the "Elephant Husbandry Resource Guide" (Olson, 2004). All observations were recorded on a data sheet (Table 4). State behaviors are those best measured by duration and used in the construction of activity budgets while event behaviors are measured by frequency and converted to rates to account for variable durations of observations. This is a common strategy used to study elephant behavior (Douglas-Hamilton et al., 2006; Evans and Harris, 2008; Foley et al., 2001; Horback et al., 2012). Elephants were selected at random for order of observations by assigning each elephant a number based upon alphabetical order $(1-13)$ and using a random number generator in Microsoft Excel. Each animal was observed three times per manipulation. Observations lasted for 10 minutes on each focal resulting in 130 minutes per observation period and 390 minutes for each manipulation. Scan sampling from the observer's left to right for the elephants' states took place before the first and seventh, and after the last observation.

\section{Dominance}

The dominance order of the elephants ascertained by elephant management personnel was used in the present study.

\section{Data Analysis}

The distance traveled by each elephant over time was calculated from the GPS devices as the Euclidean distances between successive accurate longitude and latitude 
readings measured at $5 \mathrm{sec}$ intervals (Leighty et al., 2010). The distance walked throughout the $22 \mathrm{hr}$ period was calculated by summing the walking distances of each elephant for each $22 \mathrm{hr}$ trial per treatment and averaging them for each treatment.

Behavioral data were examined by using durations and percentages for states, rates for event behaviors (frequency divided by total duration of focal length), and percentage of scans for activity budgets (Martin and Bateson, 2007). A modified Shannon-Wiener Index (Stokke and Du Toit, 2002) was used to serve as a behavioral diversity index and was calculated in the following manner: S-W Index $=-\Sigma$ $\left[\left(\mathrm{p}_{i}\right) \ln \left(\mathrm{p}_{i}\right)\right]$, where $\mathrm{p}_{i}$ is a proportion representative of the number of times a particular event behavior was performed divided by the total number of event behaviors completed, and $\Sigma$ is the sum of those proportions. Behavioral richness was calculated by counting the number of different event behaviors completed: Richness $=b$, where $b$ is the number of different behaviors complete. Behavioral richness rate was examined using the following equation: Richness rate $=\mathrm{p}_{i} /$ time visible where $\mathrm{p}_{i}$ is the number of completed event behaviors out of the number of possible event behaviors that could have been completed and time visible equates to the time the animal was observed. Evenness was calculated using the following equation: Evenness $=\mathrm{S}-\mathrm{W}$ Index/ln(r) where behavioral diversity calculated in a previous step was divided by the natural logarithm of the number of different event behaviors completed. Behavioral diversity, richness, richness rate, and evenness for each trial for each elephant were averaged to produce one value per treatment per elephant.

To assess the relationship between total distance walked and behavioral diversity as well as between total distances walked and behavioral richness and total distances 
walked and behavioral richness, a Pearson correlation was utilized (Martin and Bateson, 2007).

To investigate the role of dominance in total distance walked, behavioral diversity, and behavioral richness across the treatments, a linear regression was used between the variables mentioned above and the dominance hierarchy according to the keepers. For behavioral data, this encompassed all 13 elephants. For walking data, only the eight elephants that wore the GPS tracking anklets were compared with dominance.

As part of the behavioral data collection, social relationships between pairs of elephants were documented in the form of social touches for each elephant during their respective 10-minute continuous focal sampling. The raw numbers of social touches for each treatment both initiated and received per individual elephant were summed and analyzed as a rate per minute. A repeated measures one-way ANOVA was performed to examine potential differences in number of touches across treatments. The number of social touches from one elephant to any other were counted for each treatment using Association Indices (AI) calculated using the 'simple index' in which AI = $\mathrm{N}_{\mathrm{AB}} /\left(\mathrm{N}_{\mathrm{A}}+\mathrm{N}_{\mathrm{B}}+\mathrm{N}_{\mathrm{AB}}\right) . \mathrm{N}_{\mathrm{A}}$ and $\mathrm{N}_{\mathrm{B}}$ are indicative of the number of times either individual was seen without the other, and $\mathrm{N}_{\mathrm{AB}}$ is the total number of time that $\mathrm{N}_{\mathrm{A}}$ and $\mathrm{N}_{\mathrm{B}}$ were together (Archie et al., 2006b; Ginsberg and Young, 1992). The resulting numeric values indicative of social relationships were then mapped into sociograms using the software program Netdraw (version 2.136 [Lexington, KY, USA]).

Rates of behavioral events from the trials were averaged into their respective treatments. Specific events were combined into broader appropriate categories for data analysis. For example, the event rates for ground sniff, horizontal sniff, j-sniff, periscope 
sniff, flehmen, and vertical sniff were all combined into a category entitled

'Chemosensory events.'

To examine the effect of food on walking across treatments, distances walked $1 \mathrm{hr}$ before and $1 \mathrm{hr}$ after tossed produce and pellets were calculated. The adult male was excluded from analysis because he was separated from the herd at the time of interest. The sub-adult male was also excluded from the analysis involving tossed produce and pellets for treatment Half because he was separated from the herd at these times. A matched t-test to assess differences between the mirrored treatments was completed to determine if they could be combined for further analysis. This was the only subset of data that required the mirrored halves to be separated. Matched t-tests to look at differences at walking rates $1 \mathrm{hr}$ before and $1 \mathrm{hr}$ after tossed produce pellets were performed for each treatment.

SPSS 21.0 (Chicago, IL, USA) was used for all statistical tests. To ensure that treatments Half and Half-Mirrored as well as Both/Half and Both/Half-Mirrored could be combined into one group, a paired Wilcoxon rank sum test was completed to confirm that there were no significant differences between mirrored pairs. The lack of significant differences between mirrored treatments in any category allowed for their combination (average total walking distance: $\mathrm{P}=0.78$ and $\mathrm{P}=0.33$; average behavioral diversity: $\mathrm{P}=$ 0.70 and $\mathrm{P}=0.65$; average behavioral richness: $\mathrm{P}=0.48$ and $\mathrm{P}=0.78$ ), yielding three treatments. A Friedman's test was performed to assess the variation in average total distance walked, average behavioral diversity, average behavioral richness, and average rate of behavior for each of the event categories across treatments. 


\section{RESULTS}

\section{Walking distance}

As predicted, the three treatments had a significant effect on walking distance $(n=$ $8, \mathrm{df}=2$ chi square $=7.75, \mathrm{P}=0.02)$ with elephants walking the greatest distances when given access to both yards with food throughout (Fig. 6). However, pairwise comparisons showed no significant differences (Half - Both/Half, $\mathrm{P}=0.07$; Half - Both, $\mathrm{P}=0.07$; Both/Half - Both, $\mathrm{P}=0.21$ ), which may be due to a lack of statistical power. Total walking distances for treatment Half ranged from $5.57 \mathrm{~km}$ to $12.01 \mathrm{~km}$ with an average total distance of $7.96 \mathrm{~km}$ ( $\mathrm{SE} \pm 0.53)$. For Both/Half, walking distances ranged from 3.80 $\mathrm{km}$ to $15.46 \mathrm{~km}$ with an average of $9.45 \mathrm{~km}(\mathrm{SE} \pm 0.85)$. For the single treatment Both, walking distances ranged from $4.45 \mathrm{~km}$ to $17.4 \mathrm{~km}$ with an average of $9.77 \mathrm{~km}$ (SE \pm 0.90).

\section{Behavioral Diversity}

Behavioral diversity was greatest when elephants were provided access to both yards with food throughout, although differences were not statistically significant by treatment $(\mathrm{n}=13, \mathrm{df}=2$, chi square $=5.69, \mathrm{P}=0.06)$. The average behavioral diversity for treatment Half ranged from 1.56 to 1.94 with an average value of 1.75 ( $\mathrm{SE} \pm 0.48$ ). For Both/Half, behavioral diversity ranged from 1.43 to 2.01 with an average value of $1.79(\mathrm{SE} \pm 0.50)$. For treatment Both, behavioral diversity ranged from 1.59 to 2.11 with an average of $1.84(\mathrm{SE} \pm 0.51)$. Further examination or pairwise comparisons using a paired Wilcoxon revealed a significant difference in behavioral diversity between treatments Half and Both $(\mathrm{P}=0.03)($ Fig. 7). 


\section{Behavioral richness, richness rate, and evenness}

Behavioral evenness for treatment Half had a low of 0.74 and a high of 0.86 , with an average of $0.80(\mathrm{SE} \pm 0.01)$. Treatment Both/Half had a low of 0.68 , a high of 0.87 , and an average of $0.82(\mathrm{SE} \pm 0.02)$. Treatment Both had a low of 0.71, a high of 0.90, and an average of $0.81(\mathrm{SE} \pm 0.01)$. Behavioral richness for treatment Half had a low of 8.0, a high of 10.1, and an average of 8.84 (SE \pm 0.204 ). Richness for treatment Both/Half had a low of 8.06, a high of 10.9, and an average of 9.12 ( $\mathrm{SE} \pm 0.24)$. Treatment Both had a low of 7.2, a high of 11.9, and an average of 10.06 ( $\mathrm{SE} \pm 0.36)$. The number of behaviors performed relative to the number of possible behaviors interpreted into a richness rate did not significantly vary across treatments $(n=13, \mathrm{df}=2$, chi square $=0.45, \mathrm{P}=0.80)$. Treatment Half had a low of 0.93, a high of 1.34, and an average of 1.13 ( $\mathrm{SE} \pm 0.37$ ). Treatment Both/Half had a low of 0.84 , a high of 1.41 , and an average of 1.15 (SE \pm 0.08). Treatment Both had a low of 0.98 , a high of 1.512, and an average of 1.18 (SE \pm $0.05)$.

\section{Behavioral diversity and total distance walked}

Greater behavioral diversity was expected to correspond with a higher average distance walked since those elephants that walked were more likely to encounter greater behavioral opportunities throughout the exhibit space. The average behavioral diversity and average total distance walked were strongly related $(n=24, \mathrm{df}=22, \mathrm{R}=0.72)$ (Fig. 8). When analyzed by treatment, behavioral diversity and walking distance exhibited a strong relationship for treatment Both $(n=8, d f=6, R=0.86$, Fig. 9), and a moderate relationship for treatments Half $(n=8, d f=6, R=0.64)$ and Both/Half $(n=8, d f=6, R=$ 0.59) (the latter two are not shown). 


\section{Behavioral richness rate and total distance walked}

The rate of behavioral richness was examined to further investigate the variety of behaviors completed out of the number of behaviors possible. Without regard to treatment, walking and behavioral richness were moderately related $(\mathrm{n}=24, \mathrm{df}=22, \mathrm{R}=$ 0.54 . When analyzed by treatment, walking and behavioral richness were found to be weakly related for Half, weakly related for Both/Half, and strongly related for Both ( $\mathrm{n}=$

$8, \mathrm{df}=6, \mathrm{R}=0.48 ; \mathrm{n}=8, \mathrm{df}=6, \mathrm{R}=0.36 ;$ and $\mathrm{n}=8, \mathrm{df}=6, \mathrm{R}=0.85$, respectively) (Fig. $10)$.

\section{Behavioral evenness and total distance walked}

The distribution of behaviors and its relationship to average total distance walking was examined. Without the consideration of treatment, walking and behavioral evenness were moderately related $(\mathrm{n}=24, \mathrm{df}=22, \mathrm{R}=0.66)$. For treatment Half, walking and evenness were found to be moderately related $(n=8, d f=6, R=0.58)$. For treatment Both/Half, walking and evenness were strongly related $(n=8, d f=6, R=0.71)$. For treatment Both, walking and evenness were again strongly related $(\mathrm{n}=8, \mathrm{df}=6, \mathrm{R}=$ 0.76) (Fig. 11).

\section{Dominance and total distance walk}

Treatment Half was expected to yield greater displacements of subordinate animals in lieu of dominant ones because of the greater density of elephants compared to treatments Both/Half and Both. As the density of elephants increased, so would the likelihood of dominant animals coming into close physical proximity with subordinate animals, motivating the movement of those less dominant. For the eight elephants outfitted with GPS devices, dominance and average total distance walked were 
significantly related for treatment Half $\left(P=0.001, R^{2}=0.85\right)$, but not related for treatment Both/Half $\left(P=0.18, R^{2}=0.28\right)$ or treatment Both $\left(P=0.059, R^{2}=0.47\right)($ Fig. 12).

\section{Dominance and behavioral diversity}

Because behavioral diversity was expected to be linked to walking, it was anticipated that results from dominance and total distance walked would be very similar to dominance and behavioral diversity. Including all 13 elephants in the hierarchy, dominance and behavioral diversity were not significantly related for treatments Half ( $\mathrm{P}$ $\left.=0.274, \mathrm{R}^{2}=0.11\right)$, Both $/$ Half $\left(\mathrm{P}=0.177, \mathrm{R}^{2}=0.16\right)$, or Both $\left(\mathrm{P}=0.177, \mathrm{R}^{2}=0.16\right)$

(Fig. 13A). This lack of significance also was evident when only the 8 elephants with GPS anklets were used to determine hierarchy rankings $\left(P=0.08, R^{2}=0.432 ; P=0.10, R^{2}\right.$ $=0.38$ and $\mathrm{P}=0.41, \mathrm{R}^{2}=0.11$, respectively) (Fig. 13B).

\section{Dominance and behavioral richness rate}

Data from behavioral richness rates and dominance representative of all 13 elephants revealed that dominance and behavioral richness rate were not significantly related for treatments Half $\left(P=0.27, R^{2}=0.11\right)$, Both/Half $\left(P=0.07, R^{2}=0.27\right)$, or treatment Both $\left(P=0.47, R^{2}=0.05\right)$ (Fig. 14A). Similarly, for data pertaining to only those elephants wearing GPS anklets none of the treatments had significant relationships between behavioral richness rate and dominance $\left(P=0.13, \mathrm{R}^{2}=0.33\right.$ for treatment Half, $P=0.14, R^{2}=0.32$ for treatment Both/Half, $P=0.30, R^{2}=0.17$ for treatment Both) (Fig. 14B). 


\section{Sociality}

A greater density of elephants within treatment Half was expected to significantly increase the number of social touches among elephants and to promote stronger ties between specific individuals as evident by the simple sociality index. Neither of these hypotheses was supported. The raw number of social touches from elephant to elephant did not vary by treatment $(\mathrm{n}=13, \mathrm{df}=4$, chi square $=2.10, \mathrm{P}=0.72)$. When visually compared to one another, the sociograms resulting from the simple index calculations showed stronger ties between individuals for treatments where elephants had access to both yards. These stronger ties were particular evident among individuals belonging to the same family group (Fig. 15).

\section{Behavioral events}

If treatment impacted elephant behavior then it was suspected that there would be higher event rates of active, chemosensory, and trunk movement behaviors and lower event rates for social, exhibit perimeter interaction, and defecation/urination behaviors in treatments Both and Both/Half versus treatment Half. No significant variation among averages were found for active $(n=13, d f=2, P=0.93)$, chemosensory $(n=13, d f=2, P$ $=0.50)$, social $(n=13, d f=2, P=0.79)$, food $(n=13, d f=2, P=0.73), \operatorname{trunk}(n=13, d f$ $=2, \mathrm{P}=0.37)$, or exhibit perimeter interaction $(\mathrm{n}=13, \mathrm{df}=2, \mathrm{P}=0.79)$ behavioral events (Table 5). The most variation was present for the category Self-Maintenance ( $\mathrm{n}=$ $13, \mathrm{df}=2, \mathrm{P}=0.12)$ with significant differences between treatments Half and Both $(\mathrm{P}=$ 0.019) as well as Both/Half and Both $(\mathrm{P}=0.02)$ (Fig. 16). Unsurprisingly, active behavioral events were highest in treatment Both, but unexpectedly, so were social, selfmaintenance, and trunk movement events. Behaviors involving an interaction with the 
exhibit wall perimeter were highest in Both/Half instead of in treatment Half. Food related behaviors were highest in treatment Half as initially predicted, but so were chemosensory behaviors, which were predicted to be lowest in treatment Half.

\section{Effects of tossed produce and pellets}

Food items are often thrown into exhibit spaces, particularly when keepers cannot go into exhibit spaces with the animals. The effect of high-priority food items on walking was assessed. Encouraging movement via food introduction in the form of chopped produce and alfalfa pellets at various distributions and space was expected to be most successful in treatment Both where the elephants were given the greatest distribution of food and would presumably walk more to obtain food items. For effects of tossed produce and pellets on walking, treatments Both/Half and Both/Half-Mirrored did significantly differ $(n=6, d f=5, P=0.04)$. Significant increases in walking activity $1 \mathrm{hr}$ before and $1 \mathrm{hr}$ after tossed produce and pellets were found for treatment Both $(\mathrm{n}=7, \mathrm{df}$ $=6, P=0.0003)$ and contrary to the original hypothesis, for treatments Half $(n=6, d f=$ $5, \mathrm{P}=0.003)$ and Half-Mirrored $(\mathrm{n}=6, \mathrm{df}=5, \mathrm{P}=0.03)$ as well. As expected, treatments Both/Half $(n=7, \mathrm{df}=6, \mathrm{P}=0.41)$ and Both/Half-Mirrored $(\mathrm{n}=7, \mathrm{df}=6, \mathrm{P}=0.14) \mathrm{did}$ not have significant differences in walking distances before and after tossed produce and pellets (Fig. 17) 


\section{DISCUSSION}

For the 13 elephants housed at the San Diego Zoo Safari Park, walking distances were predicted to be greatest when both space and food distribution were increased simultaneously. The results of this study indicated that walking was highest in treatment Both, where elephants had the greatest amount of space and distribution of food, and lowest when they had the smallest amount of space with a lower distribution of food. The dominance structure of the herd was expected to influence walking distances because with greater space subordinate animals might move more freely. This hypothesis was supported in that more dominant animals walked shorter distances, and elephants in the middle of the hierarchy took the most advantage of the larger space in treatments Both/Half and Both. Behavioral values were expected to be highest when the elephants were given greater space and distribution of food. This was found for behavioral diversity, richness, and richness rate. Evenness was highest in treatment Both/Half. Behavioral values were expected to be variable due to dominance, but this was not supported by the resulting data.

Walking distances of elephants in zoological institutions have been documented using GPS units in only a few studies. The current study found an overall average of 9.06 $\mathrm{km}$ walked over a $22 \mathrm{~h}$ period. Leighty et al. (2010) reported an average of $3.68 \mathrm{~km}$ walked over a $9 \mathrm{~h}$ period at Disney's Animal Kingdom. Holdgate (2015) gathered walking distance data from 33 African and 23 Asian elephants housed at 30 different zoological institutions and calculated an average of $5.24 \mathrm{~km}$ walked over a $24 \mathrm{~h}$ period. Miller et al. (2011) reported an average of $8.65 \mathrm{~km}$ walked over a $24 \mathrm{~h}$ period at the SDZSP, which was comparable to the walking rates of African elephants in Botswana during the rainy season (Miller et al., in press). When examined across treatments, a 
significant difference in average total walking distance was determined, but not between any two given treatments. However, walking distance values for treatments Both/Half and Both were closer to one another then either was to treatment Half, providing an overall trend of higher walking rates when given a bigger space. The same trend was found at Disney's Animal Kingdom. When placed in a larger enclosure $\left(23,210 \mathrm{~m}^{2}\right)$, the adult females of the largest social grouping walked significantly more than when in smaller exhibits $\left(6,820 \mathrm{~m}^{2}\right.$ and $\left.16,390 \mathrm{~m}^{2}\right)$ (Leighty et al., 2010).

Food distribution did not appear to have a large impact on walking distances. This was surprising as food was considered the resource that would encourage movement. Previous research suggests that food spread throughout a larger space can encourage elephants to use a greater area of the exhibit (Posta et al., 2013), but that was not observed in this study. Space in and of itself may have been motivation enough for the elephants at the San Diego Zoo Safari Park, particularly since the space had secondary resources and exhibit features that the elephants might use. For example, in treatments where elephants had access to both yards, an additional pool was available. This pool would not only provide drinking water, but was also used for bathing, cooling, and play purposes. For example, the event 'spray water' occurred six times more often in treatment Both/Half (118 occurrences) than in treatment Both (20 occurrences), and almost seven times more often in treatment Both (136 occurrences) than in treatment Half (20 occurrences). Further investigation into space use may reveal effects of food distribution not evident in the present study due to the inability to investigate when and where the elephants spent their time in the exhibit. 
Behavioral data have been used in a number of studies as a method of assessing animal welfare with the standard assumption that a greater diversity of behavior lends itself to a more enriched, mentally and physically fit animal (Renner and Lussier, 2002). Behavioral richness provided some insight into differences in the number of behaviors completed in each Treatment Half, Both/Half, Both (8.84, 9.12, and 10.06 different behaviors, respectively). However, it is important to identify which behaviors are being completed in one treatment versus the other. Depending on the behaviors examined, an increase in richness may be due to an undesirable behavior, or a behavior that may not even be of interest. For example, a stereotypic behavior may be occurring in one treatment but not in another, which would increase the number of behaviors occurring and therefore richness in one of the two treatments. The elephants at the SDZSP were not seen engaging in any stereotypic behaviors during any of the observational periods. An example that would apply to this particular study is the large difference in the behavioral event "vocal", which was done 6 times more often in treatment Both (54 occurrences) than in treatment Half ( 9 occurrences). Communication is important in elephants (McComb et al., 2001), so perhaps this is a positive behavioral addition promoted by access to more space with more widely distributed food. However, the broad umbrella of vocalization for this study was categorized as any "noise emitted from the elephant in any form such as a trumpet, grumble, etc". It could be that distress, location, or aggressive calls increased, which would be more cause for concern from a behavioral and management standpoint. Interpretations such as these must be considered when determining true changes on behavior. 
The urination and defecation events are another related example. Several event behaviors were grouped together into broad categories to make statistical analysis more streamlined. Urination and defecation events were grouped together as self-maintenance. The occurrence of behaviors in the self-maintenance category were significantly greater in treatment Both than in treatments Both/Half and Half. While food distribution related to the treatment may have some impact on the rates of defecation and urination the literature reports food passage time of African elephants at varying intervals ranging from $14 \mathrm{~h}$ (Johansson, 2009) to $46 \mathrm{~h}$ (Rees, 1982). This suggests that most of the selfmaintenance behaviors recorded for this study are likely an indicator of what the elephant ate prior to the start of the trial. However, another consideration is the effect of excitement, stress, and movement on urination and defecation. Elephants walked the most in treatment Both, which had the highest levels of urination and defecation. This increase in movement could have prompted bowel and urinary tract releases. Elephants also may have encountered more stimuli with the additional resources and space in treatment Both and therefore levels of excitement and/or stress could have encouraged defecation and urination.

How often behaviors occur is another important factor, and should be considered alongside what behaviors are happening. Evenness measures the distribution of the behaviors completed. This study did not have specific target behaviors to be increased or decreased, but evenness would be particularly relevant for management programs that wish to increase or decrease particular behaviors. For example, many studies have focused on reducing stereotypic behavior and increasing the frequency of more purposeful behaviors (Anderson et al., 2010; Björk, 2011; Carlstead et al., 1991), without 
affecting the number of behaviors (richness). Yet, in this study, evenness was not affected by treatment. The greatest difference in values was between treatments Half and Both/Half at 0.018 .

Many of the behaviors animals would do in the wild become unnecessary and irrelevant in captivity (Schulte, 2000). Life in captivity removes the majority of the threats that animals face in the wild - predators, disease, starvation, drought, and the behaviors associated with these threats are no longer a necessary part of the animal's repertoire. Most animals will never or only rarely exhibit a great many natural survival behaviors once housed in captivity (Koene, 2013).

For this study, it was suspected that total distance walked and behavioral diversity, along with total distance walked and behavioral richness rate, as well as total distance walked and behavioral evenness would be significantly related as access to a larger space inherently provides greater behavioral opportunities beyond foraging (Veasy, 2006). This indicates that an animal that walks more would perform a greater variety of behaviors. For all three treatments in the current study, a strong positive relationship was evident between distance walked and behavioral diversity. Similar results were found for the behavioral richness rate, but the relationship was not as strong for treatment Both/Half. However, behavioral evenness was moderately or strongly related to walking for all three treatments. This relationship was stronger for behavioral evenness than for behavioral diversity or richness, indicating that walking had a greater impact on how many times a behavior was completed rather than what behaviors were completed. Increased walking was correlated to behavior by increasing the distribution of behavior, as well as number of behaviors completed. 
The main motivators of movement for wild elephants such as food, water, and shelter are readily provided in zoological institutions (Poole and Granuli, 2009; Posta et al., 2013). Because of this, motivators of movement for captive elephants may rely on other factors unassociated with necessities and more indicative of a zoo environment. For example, elephants may be prompted to move because of intraspecific interactions, keeper presence, guest numbers, or associations between exhibit areas and rewards. Although food may be an initial motivator, its influence on behavior may wane in comparison to factors such as sociality and dominance.

In zoos, social structures vary from those in the wild. However, dominance hierarchies still exist (Schulte, 2000). In the present study, dominance and average total walking distance were significantly related in treatment Half in that higher-ranking individuals had lower walking distances. Subordinate animals give up valuable resources and space to dominants to avoid potentially costly conflicts both in the wild (Wittemyer and Getz, 2007) and in zoos (Leighty et al., 2010). For treatments Both/Half and Both, a relationship between walking and dominance was not apparent, though the main disruption in the correlation came from the data obtained from three elephants in the middle of the dominance hierarchy who walked much greater distances in treatments Both/Half and Both than they did in treatment Half. To investigate this finding, statistics regarding dominance and walking were repeated without the three elephants in the middle. Without the three middle elephants, the opposite conclusions were reached. The relationship between walking and dominance was nonsignificant for treatment Half, but significant for treatments Both/Half and Both. Because these three elephants walked much more than their conspecifics in treatments Both/Half and Both, increasing space 
may not have the same effect in walking for all of the elephants wearing GPS anklets in this study. Rather, the elephants in the middle of the hierarchy may have taken greater advantage of the larger space than their subordinate or dominant peers. Dominance rank is known to influence space use (Murray et al., 2007). Subordinate animals are confined by the movement of other animals, particularly in a smaller space. In pigs, aggressive interactions and displacements decreased when there were a lower density of pigs presumably because subordinate animals could better control proximity of more dominant animals (Bryant and Ewbank, 1972). Dominant animals are still able to go where they desire, and they can occupy areas of their choosing. For example, a study with female chimpanzees (Pan troglodytes) found that higher ranking females occupied smaller spaces with better resources because they outcompeted their subordinate peers (Murray et al., 2007). However, in my study, walking of the elephants in the middle of the hierarchy appeared to be less controlled by dominance when they were given a larger space. This could be an anomaly of the three particular elephants in this study or it could be a generalizable pattern. However, a variable other than treatment could have impacted their walking distances. For example, the three elephants of interest consisted of a 10year-old sub-adult male, his 22-year-old mother and an unrelated 22-year-old female. During the two trials of treatment Both, I observed that the sub-adult male was actively pursuing the unrelated female, who had twice been bred by the herd's adult male. The unrelated female may have been in estrus when the two treatment Both trials took place, prompting pursuit by the sub-adult male and his mother as she tried to stay with her offspring. I did not note this activity during the trials from treatment Both/Half, but perhaps a more subtle form of the same type of activity was occurring 
Additional features available in treatments Both/Half and Both may have encouraged behaviors from subordinates who otherwise would not have utilized exhibit features because a dominant animal was already doing so, thus increasing their behavioral diversity. Greco et al.'s 2013 study at the SDZSP assessed social learning using an experimental apparatus and indicated that the most dominant member of the herd was selected as the model for the study because of a subordinate's likely apprehension to use the apparatus in her presence. Similarly, Leighty et al. (2010) found that time at the watering hole correlated positively with rank. However, in the present study, there was no relationship between overall behavioral diversity and dominance or overall behavioral richness and dominance for any of the treatments for the eight elephants wearing GPS devices or for all 13 elephants, indicating that dominance did not affect the behaviors examined in this study.

In the present study, social interactions did not significantly differ from one treatment to another. However, a visual assessment via a sociogram of social relationships indicated stronger ties between individuals, particularly those belonging to the same family groups, when elephants had the larger amount of space, regardless of food distribution. The elephants at the SDZSP may maintain closer proximity to family groups when in a larger space and thereby increase social interactions. Elephants have strong family ties, and are protective of their young (Lee, 1987; Archie et al., 2006b). When in a smaller space, mothers/allomothers may be better able to scan the area for dangers and potential threats. However, when given both sides of the exhibit, these threats and dangers may not be perceived as quickly, potentially resulting in a greater 
likelihood that calves and mothers/allomothers would stay within closer physical proximity resulting in more frequent social behaviors.

Concern for elephant welfare in captive facilities has prompted a number of exhibit and management changes, including those surrounding enrichment programs. Knowledge of how multiple enrichment types affect elephant behavior and walking is crucial. At the SDZSP, varying space and food distribution had a significant effect on the total distance walked, but this was variable among individuals. This variance was likely due to dominance, as three of the middle-placed dominant animals increased their walking in treatments Both/Half and Both more than elephants of higher or lower rank. Zoos housing a larger number of elephants with a clear high, middle, and low dominance hierarchy may be able to apply a similar method of providing more space to increase the amount of walking by their middle dominant elephants. Because dominance and behavior were not related in any of the treatments, it is unlikely that the hierarchy exhibited at the SDZSP affected the behaviors of any particular individual or dominance grouping, unlike walking. Behaviorally, effects of treatment varied statistically depending on the measure used. According to the modified S-W Index, elephants at the SDZSP were significantly more behaviorally diverse in treatment Both than in treatment Half, though statistics regarding richness rates did not reflect these same conclusions, indicating that evenness may have had a greater impact on behavioral diversity than expected. Evenness of behaviors alone did not vary across treatments. However, richness values were highest in treatment Both, and the overall trend of greater behavioral diversity as represented by behavioral diversity, richness rate, and richness was still apparent. Other zoos housing elephants may see the number of behaviors completed out of an assigned repertoire 
increase when more space is provided and food is more widely distributed, but it is imperative that the individual behaviors being completed are examined and deemed appropriate before a positive connotation is associated with the result. Additionally, thel absence of certain behaviors should be evaluated in terms of behavioral opportunity; just because a behavior is commonly performed in the wild does not mean it is necessary or even desired in captivity. Each individual elephant's behavior should be of interest when assessing the impacts of exhibit and enrichment changes as a "one-size-fits all" approach may be futile (Swaisgood and Shepherdson, 2005). For example, Elzankowski and Sergiel (2006) found that changes to the management routine enhanced undesirable stereotypic behavior in a female Asian elephant. In the current study, walking and the behavioral measures were, for the most part, strongly related. Zoos seeking changes in the behavior of their elephants may benefit from taking steps to increase walking distances. For example, social touching between elephant pairs increased with walking. Finally, elephants at the SDZSP may have learned the routine and placement of items over time, as evidenced with the tossed produce and pellets in treatment Both/Half. Habituation may decrease the positive effects of enrichment, thus reducing their movement and behaviors. Randomized schedules (Murphy et al., 2003; Quirke and O'Riodan, 2011; Tarou and Bashaw, 2007) with longer time gaps in between enrichment items (Anderson et al., 2010; Murphy et al., 2003; Tarou and Badsaw, 2007), and greater variety of items (Holdgate, 2015) may provide the best method of increasing the effectiveness of enrichment practices.

The housing of animals in captivity brings with it the responsibility to care for their physical and mental well-being. The practice of enrichment goes beyond meeting 
the necessities of maintaining life; however, the success of enrichment requires a clear set of objectives and means of assessment. Early uses of enrichment focused on reducing undesired behaviors (i.e., stereotypies), but today enrichment strives to achieve a more holistic level of animal welfare. Thus, it seems reasonable that multiple forms of assessment are needed to determine the overall value of an enrichment program for a particular set of animals. The present study was the first to use a Shannon-Wiener Index as a measure of behavioral diversity for elephants. This index has had limited use in other species but based on the results herein, it seems beneficial to extend its use as an added tool for assessing enrichment practices. In addition, direct measures such as walking rates, spatial use of the exhibit, social interactions, and the frequency and type of behaviors exhibited are meaningful measures for evaluating the success of enrichment. The present study used a number of these measures to show that greater exhibit space and a wider distribution of food without increasing the amount of food delivered some benefit to the elephants at SDZSP in the form of increased total walking. The space and food manipulations did not have a profound influence on elephant behavior, suggesting that other forms of enrichment may be needed to alter behavioral repertoires. Through well controlled, extensive experimental studies like the one performed at the SDZSP, more can be learned about the value of enrichment practices to enhance the welfare of elephants and other animals housed at zoological facilities. 
Table 1. A matrix of the predictions of outcomes for this particular study in relevance to each treatment, the variables walking and behavior, and how dominance was expected to impact those variables.

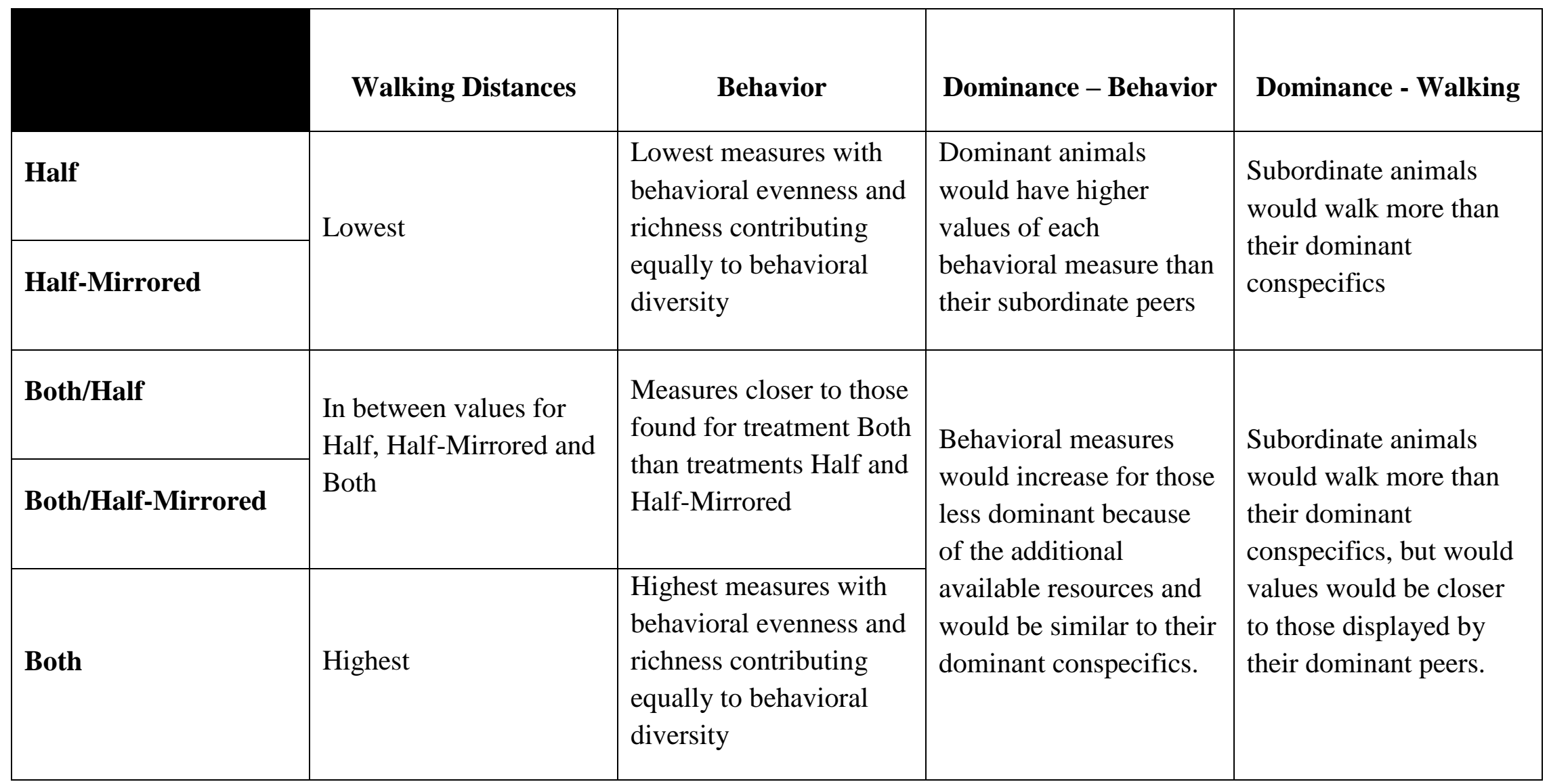


Table 2. Information about the African elephants at the San Diego Zoo Safari Park.

\begin{tabular}{|c|c|c|c|c|c|}
\hline Name & Gender & $\begin{array}{l}\text { Study } \\
\text { Number }\end{array}$ & $\begin{array}{l}\text { Birth } \\
\text { Year }\end{array}$ & $\begin{array}{l}\text { Identification } \\
\text { characteristics as of } \\
\text { March } 2014\end{array}$ & Relations \\
\hline Swazi & Female & 2 & 1991 & $\begin{array}{l}\text { The largest female } \\
\text { of the herd. Has a } \\
\text { ventral oedema. }\end{array}$ & $\begin{array}{l}\text { Mother to eMachembe } \\
\text { and Qinisa. }\end{array}$ \\
\hline Umngani & Female & 4 & 1990 & $\begin{array}{l}\text { The female with the } \\
\text { longest tusks. }\end{array}$ & $\begin{array}{l}\text { Mother of Khosi, } \\
\text { Ingadze, and } \\
\text { Inhlonipho. }\end{array}$ \\
\hline Msholo & Male & 1 & 1990 & $\begin{array}{l}\text { The largest bull } \\
\text { elephant. }\end{array}$ & $\begin{array}{l}\text { No paternal relations, } \\
\text { but often pursues } \\
\text { Umngani for mating. }\end{array}$ \\
\hline Vus'musi & Male & 5 & 2004 & $\begin{array}{l}\text { Has one short tusk } \\
\text { and one long tusk } \\
\text { with broken end. }\end{array}$ & $\begin{array}{l}\text { First baby born to the } \\
\text { herd. } \\
\text { Mother is Ndula. } \\
\text { Friends with Msholo. }\end{array}$ \\
\hline Ndulamitsi & Female & 3 & 1990 & $\begin{array}{l}\text { Tallest female, with } \\
\text { a right tusk that curls } \\
\text { underneath. }\end{array}$ & $\begin{array}{l}\text { Mother of Vus'musi } \\
\text { and Lutsandvo. } \\
\text { Was pregnant with } \\
\text { Vus'musi when she } \\
\text { arrived from Africa. }\end{array}$ \\
\hline Khosi & Female & 6 & 2006 & $\begin{array}{l}\text { Has a tusk with a } \\
\text { stainless steel cap on } \\
\text { the end. }\end{array}$ & $\begin{array}{l}\text { Daughter of Mabu and } \\
\text { Umngani. } \\
\text { Older sister of Ingadze } \\
\text { and Inhlonipho. }\end{array}$ \\
\hline Phakamile & Female & 7 & 2007 & $\begin{array}{l}\text { Has short, stubby, } \\
\text { flattened tusks. }\end{array}$ & $\begin{array}{l}\text { Daughter of Umoya } \\
\text { and Mabu } \\
\text { Big sister to Emanti. }\end{array}$ \\
\hline
\end{tabular}




\begin{tabular}{|c|c|c|c|c|c|}
\hline \multicolumn{6}{|c|}{ Table 1 continued } \\
\hline Ingadze & 9 & Male & 2009 & $\begin{array}{l}\text { Tusk emerge from } \\
\text { an obtuse angle. }\end{array}$ & $\begin{array}{l}\text { Son of Umngani and } \\
\text { Mabu. } \\
\text { Brother to Inhlonipho } \\
\text { and Khosi. }\end{array}$ \\
\hline Lutsandvo & 8 & Male & 2010 & The largest calf. & $\begin{array}{l}\text { Son of Ndlula and } \\
\text { Mabu. }\end{array}$ \\
\hline eMacembe & 10 & Male & 2010 & $\begin{array}{l}\text { Grayer in color than } \\
\text { the other calves. }\end{array}$ & $\begin{array}{l}\text { Son of Swazi and } \\
\text { Mabu. } \\
\text { Big brother to sister } \\
\text { Qinisa. }\end{array}$ \\
\hline Emanti & 11 & Male & 2010 & $\begin{array}{l}\text { Missing one tusk, } \\
\text { relatively small for } \\
\text { his age. }\end{array}$ & $\begin{array}{l}\text { Son of Umoya and } \\
\text { Mabu } \\
\text { Born in main yard. } \\
\text { Younger brother to } \\
\text { Phakamile. }\end{array}$ \\
\hline Inhlonipho & 12 & Male & 2011 & Has milk tusks. & $\begin{array}{l}\text { Son of Umngani and } \\
\text { Mabu. } \\
\text { Little brother to Khosi } \\
\text { and big brother to } \\
\text { Ingadze. } \\
\text { Currently the youngest } \\
\text { boy. }\end{array}$ \\
\hline Qinisa & 13 & Female & 2012 & $\begin{array}{l}\text { Smallest calf, tail } \\
\text { hairs often sparse. }\end{array}$ & $\begin{array}{l}\text { Newest herd member. } \\
\text { Daughter of Swazi and } \\
\text { Mabu. } \\
\text { Little sister to Mac. }\end{array}$ \\
\hline
\end{tabular}


Table 3. The data sheet used to record behavioral data in the form of all occurrence events and continuous states as well as scan sampling.

\section{Date: Time: Temp.: Subject:}

\begin{tabular}{|c|c|c|c|c|}
\hline Event & Occurrences & Event & \multicolumn{2}{|c|}{ Occurrences } \\
\hline Be Pushed & & Self- Touch & & \\
\hline Collecting Food & & Shake Food & & \\
\hline Defecate & & Social Touch & Touch & $\begin{array}{c}\text { Touched } \\
\text { By }\end{array}$ \\
\hline Dust & & Spray Water & & \\
\hline Dig & & Steal & & \\
\hline Drink & & Throw Food & & \\
\hline Enrichment Use & & Throw Other & & \\
\hline Flehmen & & Trunk Curl & & \\
\hline Food Hoard & & Trunk Flick & & \\
\hline Ground Sniff & & Trunk Swing & & \\
\hline Head Shake & & Trunk Wriggle & & \\
\hline Horizontal Sniff & & Urinate & & \\
\hline J Sniff & & Vertical Sniff & & \\
\hline $\begin{array}{l}\text { Perimeter } \\
\text { Interaction }\end{array}$ & & Vocalization & & \\
\hline Periscope Sniff & & Other: & & \\
\hline Push & & & & \\
\hline Scratch & & & & \\
\hline
\end{tabular}

\begin{tabular}{|c|l|l|l|l|l|l|l|l|l|l|l|l|}
\hline $\begin{array}{c}\text { Time } \\
(\mathrm{min} ., \mathrm{sec} .)\end{array}$ & & & & & & & & & & & & \\
\hline State & & & & & & & & & & & & \\
\hline
\end{tabular}

\begin{tabular}{|l|c|c|c|c|c|c|c|c|c|c|c|c|c|c|}
\hline $\begin{array}{l}\text { Scan } \\
\text { (left to right) }\end{array}$ & ID & & & & & & & & & & & & & \\
\hline & State & & & & & & & & & & & & & \\
\hline
\end{tabular}


Table 4. The ethogram utilized to define behaviors. States are italicized; events are in regular font.

\begin{tabular}{|c|c|}
\hline \multicolumn{2}{|l|}{ Water Related } \\
\hline Behavior & Description \\
\hline Drink (DR) & $\begin{array}{l}\text { Obtaining water from a receptacle, pool, or hose and placing } \\
\text { into the mouth. }\end{array}$ \\
\hline In water $\left(\mathrm{H}_{2} \mathrm{O}\right)$ & $\begin{array}{l}\text { At least } 25 \% \text { of the body is submerged in the water and play } \\
\text { is not occurring. }\end{array}$ \\
\hline Spray (SPR) & $\begin{array}{l}\text { Water is sucked into the elephant's trunk and is ejected back } \\
\text { out of the trunk with force on self, another elephant, or into } \\
\text { nearby space. }\end{array}$ \\
\hline Water Play $\left(\mathrm{H}_{2} \mathrm{OP}\right)$ & $\begin{array}{l}\text { Various behaviors, such as swimming, splashing, skimming, } \\
\text { submerging and the elephant is not engaging with another } \\
\text { elephant. }\end{array}$ \\
\hline
\end{tabular}

\begin{tabular}{|c|c|}
\hline \multicolumn{2}{|l|}{ Trunk Related } \\
\hline Behavior & Description \\
\hline $\begin{array}{l}\text { Self-Directed Touch } \\
\text { (SDT) }\end{array}$ & $\begin{array}{l}\text { An elephant touches itself anywhere on its body with the tip } \\
\text { of its own trunk. }\end{array}$ \\
\hline Trunk Curl (TC) & The bottom third of the trunk curls in any direction. \\
\hline Trunk Flick (TF) & $\begin{array}{l}\text { The very end of the trunk just prior to the trunk's finger like } \\
\text { projections moves back and forth. Trunk is generally in a } \\
\text { downward position. }\end{array}$ \\
\hline Trunk Swing (TS) & $\begin{array}{l}\text { The entire trunk is moved back and forth in any direction, } \\
\text { sometimes including a head bobbing motion. }\end{array}$ \\
\hline Trunk Wriggle (TW) & $\begin{array}{l}\text { The trunk is lifted at least parallel to the ground if not higher, } \\
\text { folds upon itself, and is then dropped. }\end{array}$ \\
\hline
\end{tabular}

\begin{tabular}{|l|l|}
\hline Investigation Related & Description \\
\hline Behavior & $\begin{array}{l}\text { The trunk tip is placed over the paired openings to the } \\
\text { vomeronasal organ located in the roof of the mouth. }\end{array}$ \\
\hline Flehmen (FL) & $\begin{array}{l}\text { The trunk is placed just above the ground but does not make } \\
\text { contact; it moves slightly side to side. }\end{array}$ \\
\hline Ground Sniff (GS) & $\begin{array}{l}\text { The trunk is extended parallel or within 45 degrees of being } \\
\text { parallel to the ground, reaching in front of the elephant. }\end{array}$ \\
\hline Horizontal Sniff (HS) & $\begin{array}{l}\text { The bottom half of the trunk is curved to either side in the } \\
\text { shape of the letter 'J'. }\end{array}$ \\
\hline Periscope Sniff (PS) & $\begin{array}{l}\text { The end portion of the trunk is placed directly on top of the } \\
\text { head in a salute position. }\end{array}$ \\
\hline Vertical Sniff (VS) & $\begin{array}{l}\text { The trunk is extended upwards reaching over the head and is } \\
\text { not reaching for a physical object. }\end{array}$ \\
\hline
\end{tabular}




\begin{tabular}{|l|l|}
\hline Food Related & Description \\
\hline Behavior & $\begin{array}{l}\text { Organizing food within one trunk's length reach into a pile } \\
\text { that can be picked up as one unit and placed into mouth. } \\
\text { Usually done with sparse pieces of hay or alfalfa pellets. }\end{array}$ \\
\hline Follecting Food (CF) Hoarding (FH) & $\begin{array}{l}\text { Storing more than one mouthful of food on top of body, } \\
\text { head, wedged against tusk(s) or folded in trunk and } \\
\text { removing the food matter at least one body length from the } \\
\text { initial site. }\end{array}$ \\
\hline Eating Browse $(E B)$ & Eating branches, bushes, bark, and/or other leafy plants. \\
\hline Eating Hay $(E H)$ & Eating hay without combining the hay with browse. \\
\hline Eating Other $(E O)$ & $\begin{array}{l}\text { Eating food that cannot be classified as browse or hay. Such } \\
\text { food includes beet pulp, alfalfa pellets, mud, dirt, sand, } \\
\text { stones, salt, etc. }\end{array}$ \\
\hline Shake Food (SF) & $\begin{array}{l}\text { The elephant shakes the food already held in its trunk with a } \\
\text { force large enough to break up this food matter. }\end{array}$ \\
\hline Throw Food (THF) & $\begin{array}{l}\text { An elephant throws, flings, or tosses the food matter without } \\
\text { eating any remnants that the elephant may have held onto } \\
\text { during the throwing action. }\end{array}$ \\
\hline
\end{tabular}

\begin{tabular}{|l|l|}
\hline Non-Locomotion Related \\
\hline Behavior & Description \\
\hline Laying Down (LD) & $\begin{array}{l}\text { At least } 75 \% \text { of the body is on the ground with limited to no } \\
\text { movement. }\end{array}$ \\
\hline Standing (S) & $\begin{array}{l}\text { Elephant is elevated on all four legs with little to no leg } \\
\text { movement and is not eating, drinking, or nursing. }\end{array}$ \\
\hline
\end{tabular}

\begin{tabular}{|c|c|}
\hline \multicolumn{2}{|l|}{ Social Contact Related } \\
\hline Behavior & Description \\
\hline Be Pushed (BP) & $\begin{array}{l}\text { Receive force from another elephant's body that results in } \\
\text { the physical displacement of the subject elephant. }\end{array}$ \\
\hline Push (P) & $\begin{array}{l}\text { Exert force on the body of another elephant to physically } \\
\text { displace it. }\end{array}$ \\
\hline Social Play $(S P)$ & $\begin{array}{l}\text { Engaging in friendly lively contact out of the water with at } \\
\text { least one other elephant without eating. Could include } \\
\text { sparring, enrichment use, and the like. }\end{array}$ \\
\hline $\begin{array}{l}\text { Social Play in Water } \\
\left(\mathrm{H}_{2} \mathrm{OSP}\right)\end{array}$ & $\begin{array}{l}\text { The elephant is in the water and it is playing with at least } \\
\text { one other elephant that is also in the water. }\end{array}$ \\
\hline Social Touch (ST) & $\begin{array}{l}\text { Head, trunk, tusk, or body contact with another elephant that } \\
\text { does not lead to play, aggression, or physical displacement. }\end{array}$ \\
\hline Steal (ST) & $\begin{array}{l}\text { An elephant takes an object (food, enrichment device, etc.) } \\
\text { within one trunk's length of another elephant. }\end{array}$ \\
\hline Vocalization (V) & $\begin{array}{l}\text { An elephant emits a vocal noise in any form such as a } \\
\text { trumpet, grumble, etc. }\end{array}$ \\
\hline
\end{tabular}




\begin{tabular}{|l|l|}
\hline Object Related \\
\hline Behavior & Description \\
\hline $\begin{array}{l}\text { Enrichment Use (non- } \\
\text { food) (EU) }\end{array}$ & $\begin{array}{l}\text { Active participation with any enrichment device that is not } \\
\text { associated with food. Such devices could include barrels, } \\
\text { artificial rock surfaces, artificial tree stumps, etc. }\end{array}$ \\
\hline
\end{tabular}

\begin{tabular}{|l|l|}
\hline \multicolumn{2}{|l|}{ Self-Maintenance Related } \\
\hline Behavior & Description \\
\hline Defecate (DEF) & Discharging dung. \\
\hline Urine (U) & Passing a stream of urine. \\
\hline
\end{tabular}

\begin{tabular}{|l|l|}
\hline Calf Related \\
\hline Behavior & Description \\
\hline Nursing $(N)$ & $\begin{array}{l}\text { (Adult) Mother stands for calf to suckle or (Calf) calf suckles } \\
\text { mother to receive milk. }\end{array}$ \\
\hline
\end{tabular}

\begin{tabular}{|c|c|}
\hline \multicolumn{2}{|l|}{ Locomotion Related } \\
\hline Behavior & Description \\
\hline Dig (DG) & $\begin{array}{l}\text { The elephant stabs tusks into ground to displace dirt, sand, } \\
\text { gravel, or other material. May also use trunk or foot. }\end{array}$ \\
\hline Dust (D) & $\begin{array}{l}\text { The trunk's fingers pick up dust and fling it onto the } \\
\text { elephant's top, side, or underbelly. }\end{array}$ \\
\hline Head Shake (HSH) & $\begin{array}{l}\text { The elephant shakes its head in one successive movement so } \\
\text { as to remove debris, give a social cue, etc. }\end{array}$ \\
\hline Scratch $(\mathrm{SC})$ & $\begin{array}{l}\text { Rub head, foot, or body back and forth against self, wall, } \\
\text { rock, tree, or other large object. }\end{array}$ \\
\hline Throw Other (TO) & $\begin{array}{l}\text { An elephant throws, flings, or tosses an object or mud onto } \\
\text { itself, others, or into nearby space. }\end{array}$ \\
\hline Walking $(W)$ & $\begin{array}{l}\text { Moving to produce a walking gait without simultaneously } \\
\text { eating, drinking, or playing. }\end{array}$ \\
\hline $\begin{array}{l}\text { Walking and Eating } \\
\text { Browse (WEB) }\end{array}$ & $\begin{array}{l}\text { The elephant is moving to produce a walking gait and is } \\
\text { simultaneously eating browse. Browse could consist of } \\
\text { branches, bushes, bark, and/or other leafy plants. }\end{array}$ \\
\hline $\begin{array}{l}\text { Walking and Eating Hay } \\
(\text { WEH) }\end{array}$ & $\begin{array}{l}\text { The elephant is moving to produce a walking gait and is } \\
\text { simultaneously eating hay. }\end{array}$ \\
\hline $\begin{array}{l}\text { Walking and Eating } \\
\text { Other (WEO) }\end{array}$ & $\begin{array}{l}\text { The elephant is moving to produce a walking gait and is } \\
\text { simultaneously eating food that cannot be classified as } \\
\text { browse or hay. Such food includes beet pulp, alfalfa pellets, } \\
\text { mud, dirt, sand, stones, salt, etc. }\end{array}$ \\
\hline Wallowing (WA) & $\begin{array}{l}\text { Lying down and moving body back and forth to cover the } \\
\text { body in mud, dirt, or sand. }\end{array}$ \\
\hline
\end{tabular}




\begin{tabular}{|l|l|}
\hline \multicolumn{2}{|l|}{ Zoological Institution Related } \\
\hline Behavior & Description \\
\hline Loitering $(L)$ & $\begin{array}{l}\text { The elephant is standing in an exhibit within two body lengths } \\
\text { of a closed gate for longer than } 30 \text { seconds and is not eating, } \\
\text { drinking, playing or nursing. }\end{array}$ \\
\hline Perimeter Interaction (PI) & $\begin{array}{l}\text { An elephant approaches the perimeter of the exhibit and } \\
\text { investigates but does not use the perimeter for other purposes } \\
\text { such as scratching. }\end{array}$ \\
\hline Keeper Interaction $(K I)$ & $\begin{array}{l}\text { The elephant is within 20 feet of a human handler and is } \\
\text { either accepting directions from, interacting with, or } \\
\text { loitering around, the individual. Such interaction could } \\
\text { occur during training sessions, herd shifting, or positive } \\
\text { reinforcement opportunities. }\end{array}$ \\
\hline
\end{tabular}

\begin{tabular}{|l|l|}
\hline Other Related \\
\hline Behavior & Description \\
\hline Not Visible $(N V)$ & The elephant is out of sight from the observer. \\
\hline Other / Additional Notes & $\begin{array}{l}\text { Any other events that may be of interest to the study but were } \\
\text { not included in the ethogram. }\end{array}$ \\
\hline
\end{tabular}

\begin{tabular}{|l|l|}
\hline Covariant & $\begin{array}{l}\text { One or more human handlers is within sight or can be heard } \\
\text { by the elephant but s/he is not actively engaging with the } \\
\text { elephant in any kind of training, reward acceptance, or } \\
\text { shifting activity. }\end{array}$ \\
\hline Keeper Interaction & $\begin{array}{l}\text { One or more of the human handlers is actively engaged with } \\
\text { the elephant. This includes rewarding, training, general } \\
\text { husbandry practices, or shifting. }\end{array}$ \\
\hline
\end{tabular}


Table 5. The p-values associated with various behavioral event rate categories across treatments. Significant values across treatments were found using a Friedman test and a paired Wilcoxon was used to assess differences between any two given treatments. All statistics were ran with $\mathrm{n}=13$ and $\mathrm{df}=2$.

\begin{tabular}{|l|l|l|}
\hline Behavioral Event Category & P-Value & $\begin{array}{l}\text { Chi } \\
\text { Square }\end{array}$ \\
\hline Active & 0.93 & 0.15 \\
\hline Chemosensory & 0.5 & 1.39 \\
\hline Social & 0.80 & 0.46 \\
\hline Food & 0.74 & 0.62 \\
\hline Trunk Movement & 0.37 & 2 \\
\hline Exhibit Perimeter Interaction & 0.80 & 0.46 \\
\hline $\begin{array}{l}\text { Self-Maintenance } \\
\text { Between Both and Half } \\
\text { Between Both/Half and Half }\end{array}$ & 0.11 & $0.02^{*}$ \\
$0.02^{*}$ & \\
\hline
\end{tabular}

$* \mathrm{P}<0.05$ 




Fig. 1 As of November 2015, the most recent Google Earth image of the two African elephant enclosures and their connection via a hydraulic gate at the San Diego Zoo Safari Park in Escondido, Ca. Image taken from Google Earth. Text provided by C. Hacker. 


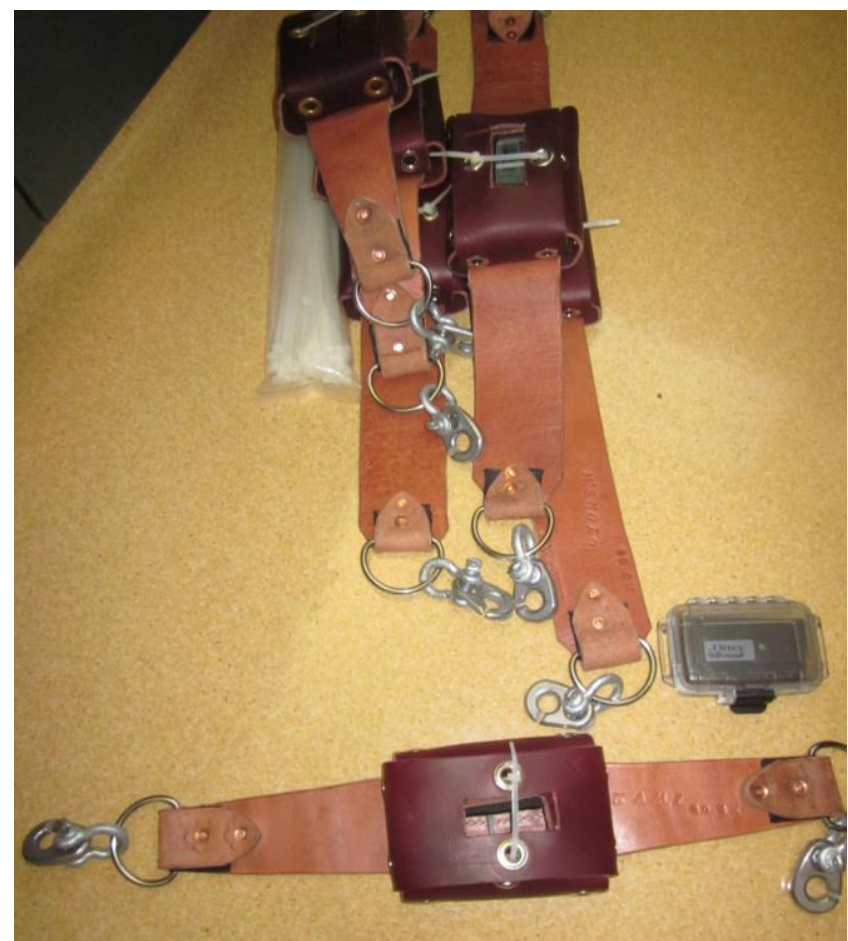

Fig. 2 The GPS anklets used to assess $22 \mathrm{~h}$ walking rates. A GPS tracking unit (Qstarz BT-Q1000X [Taipei, Taiwan]) was encased in a waterproof Otterbox, ${ }^{\mathrm{TM}}$ which was then secured in a leather pouch in the middle of the anklet. Photo credit: C. Hacker 

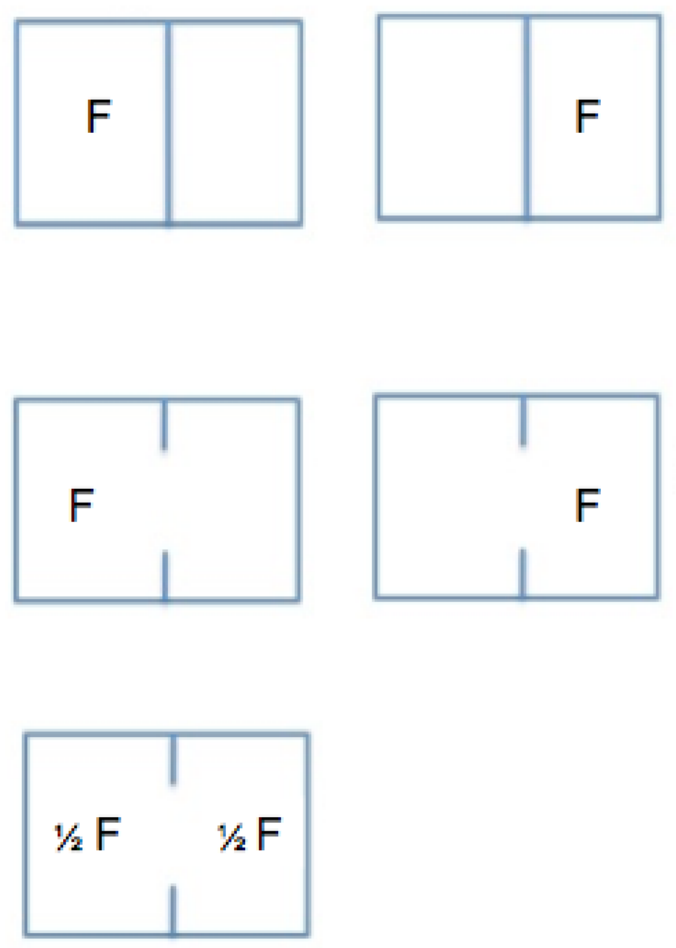

Fig. 3 Exhibit treatments of space and food $(F)$. The top row shows treatment Half (access to one yard of the exhibit with food in that yard, see Fig. 1) and its mirrored counterpart, Half-Mirrored. The second row shows treatment Both/Half (access to both yards of the exhibit with food in one yard) and its mirrored counterpart, Both/HalfMirrored. The bottom row show treatment Both (access to both yards of the exhibit with food in both). The $1 / 2$ is to clarify that the same amount of total food (F) was used in treatment Both, but was distributed over a greater area. 


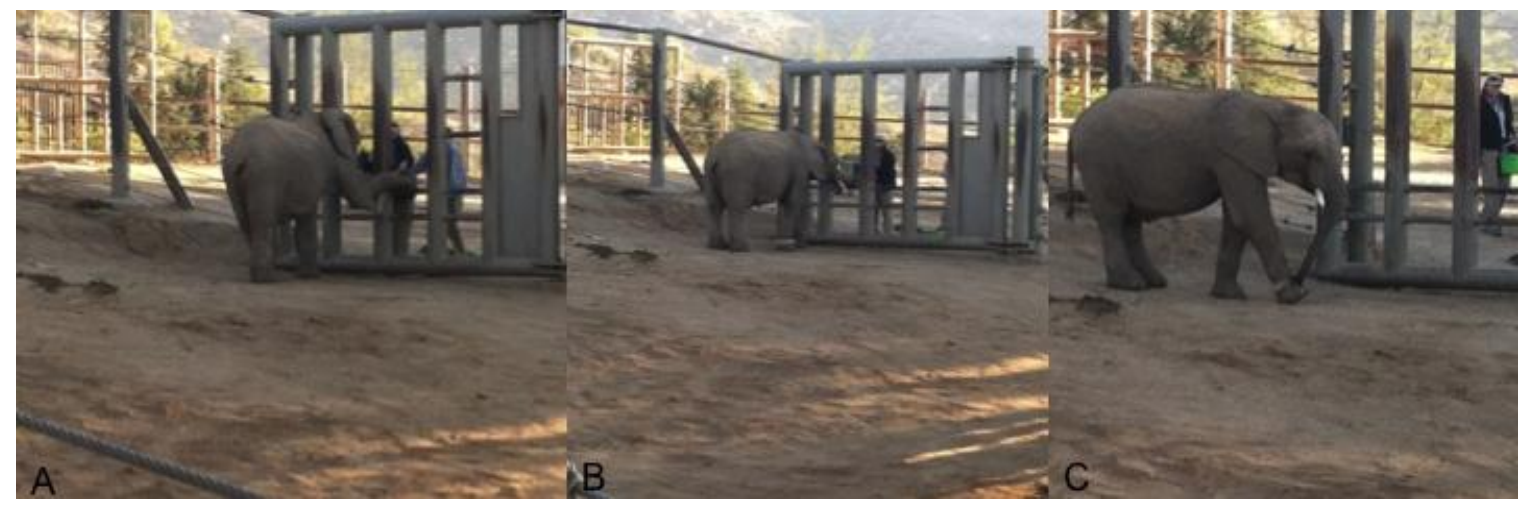

Fig. 4A, 4B, and 4C A) Gadze, a 4-year-old male, presents his right front foot to two members of the elephant management staff to receive a training anklet. B) Upon cooperation and acceptance of the anklet, Gadze received alfalfa pellets and praise. C) Gadze was permitted to roam the elephant yard and investigate his anklet. Photo credit: C. Hacker 


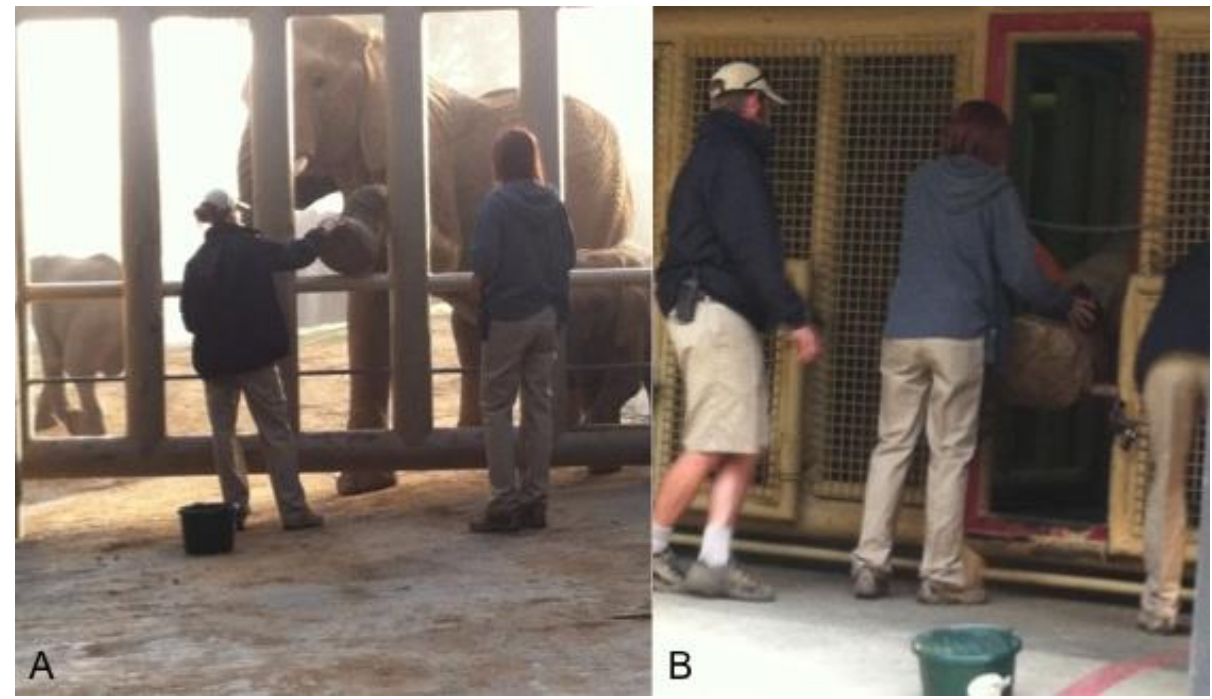

Fig. 5A and 5B A) The anklet design was tested on Swazi, the dominant female of the herd. B) The anklet design was tested on M'sholo, the bull elephant. Testing of the anklets ensured that they could be safely attached and removed, and that they did not provide any visible discomfort. Photo credit: C. Hacker. 


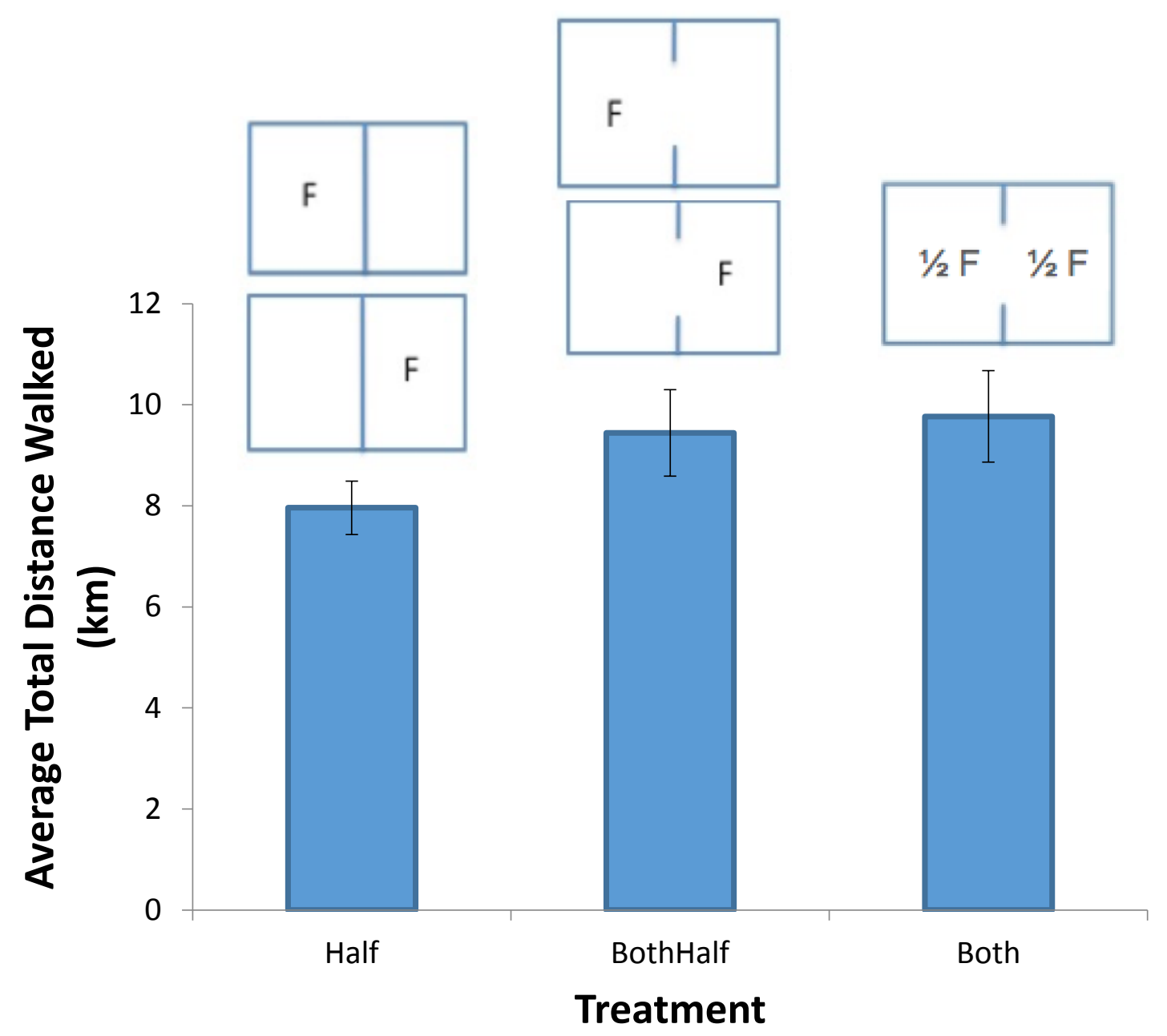

Fig. 6 The average total walking distance for the entire $22 \mathrm{hr}$ period across the different treatments of space and food. A significant difference among treatments was found $(\mathrm{n}=$ $8, \mathrm{df}=2$, chi square $=7.75, \mathrm{P}=0.021)$, but significance between any given pair was not evident. Bars are \pm 1 SEM. 


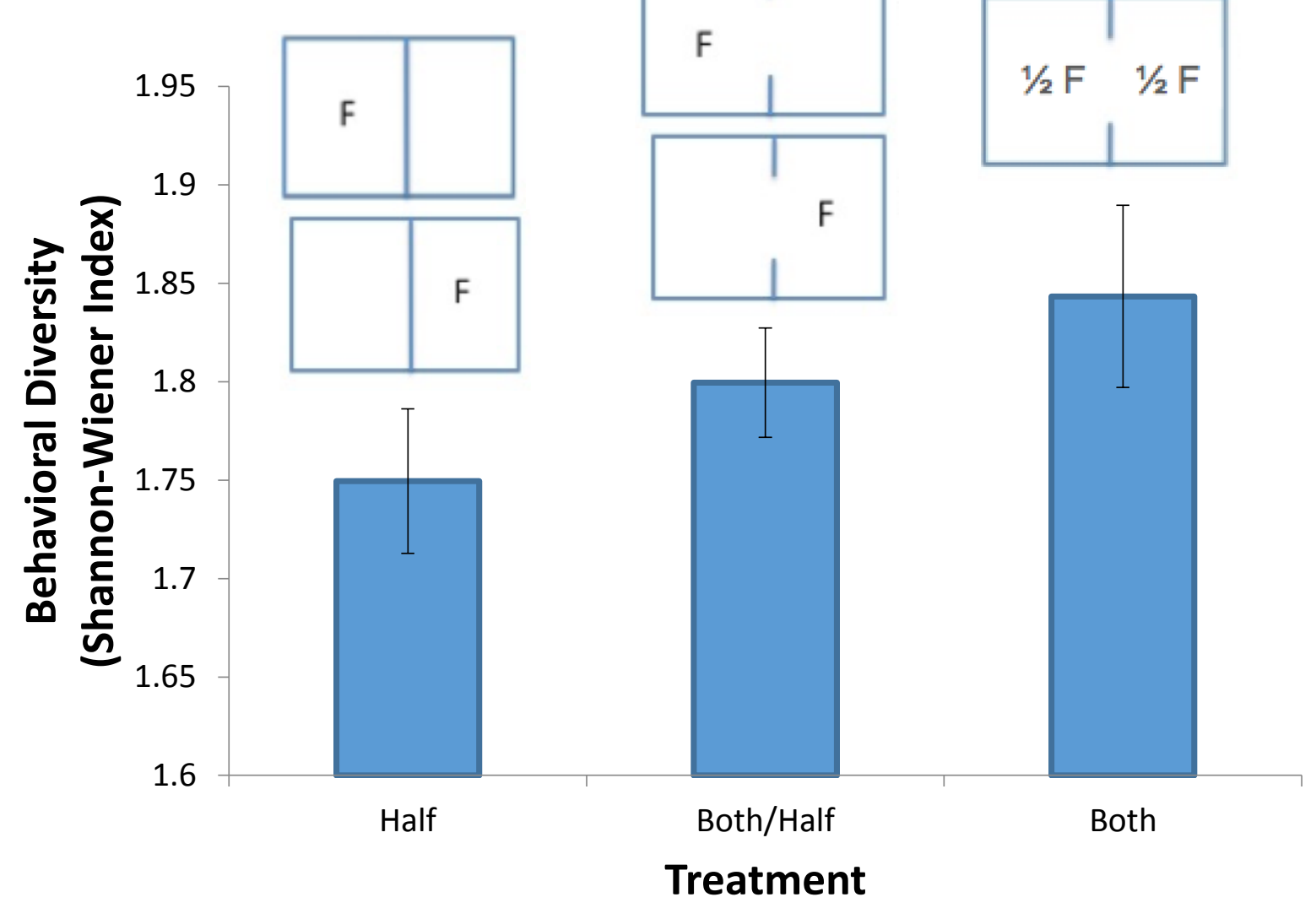

Fig. 7 The average Shannon-Wiener Index across the different treatments of space and food. A significant difference between treatments Half and Both was found $(\mathrm{n}=13, \mathrm{df}=$ 2, chi square $=5.69, \mathrm{P}=0.028)$. Bars are \pm 1 SEM. 


\section{All Treatments}

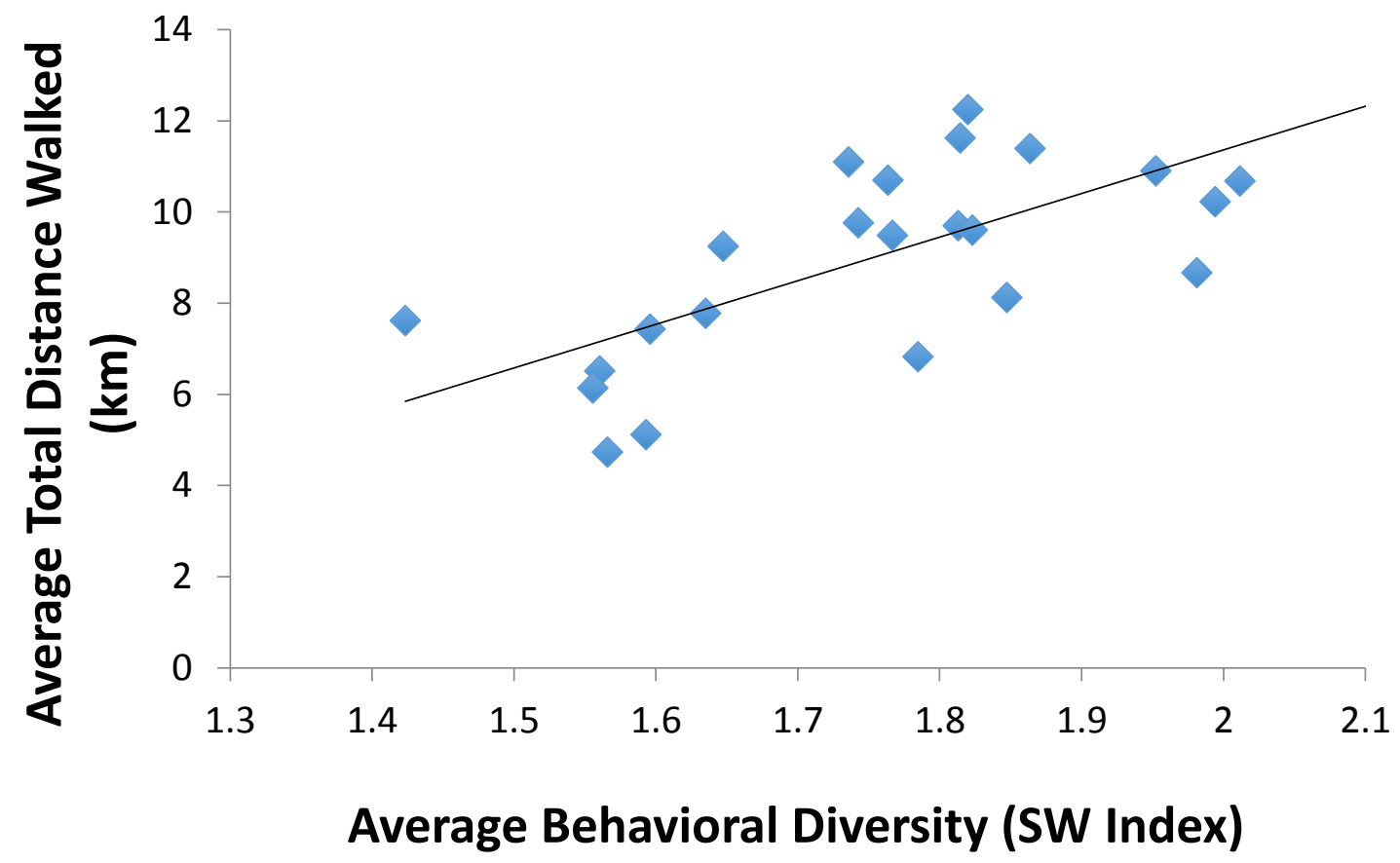

Fig. 8 The average behavioral diversity and average total distance walked for the $22 \mathrm{hr}$ treatment period. This relationship was strongly related when all treatments were considered $(\mathrm{R}=0.72)$. 


\section{Treatment Both}

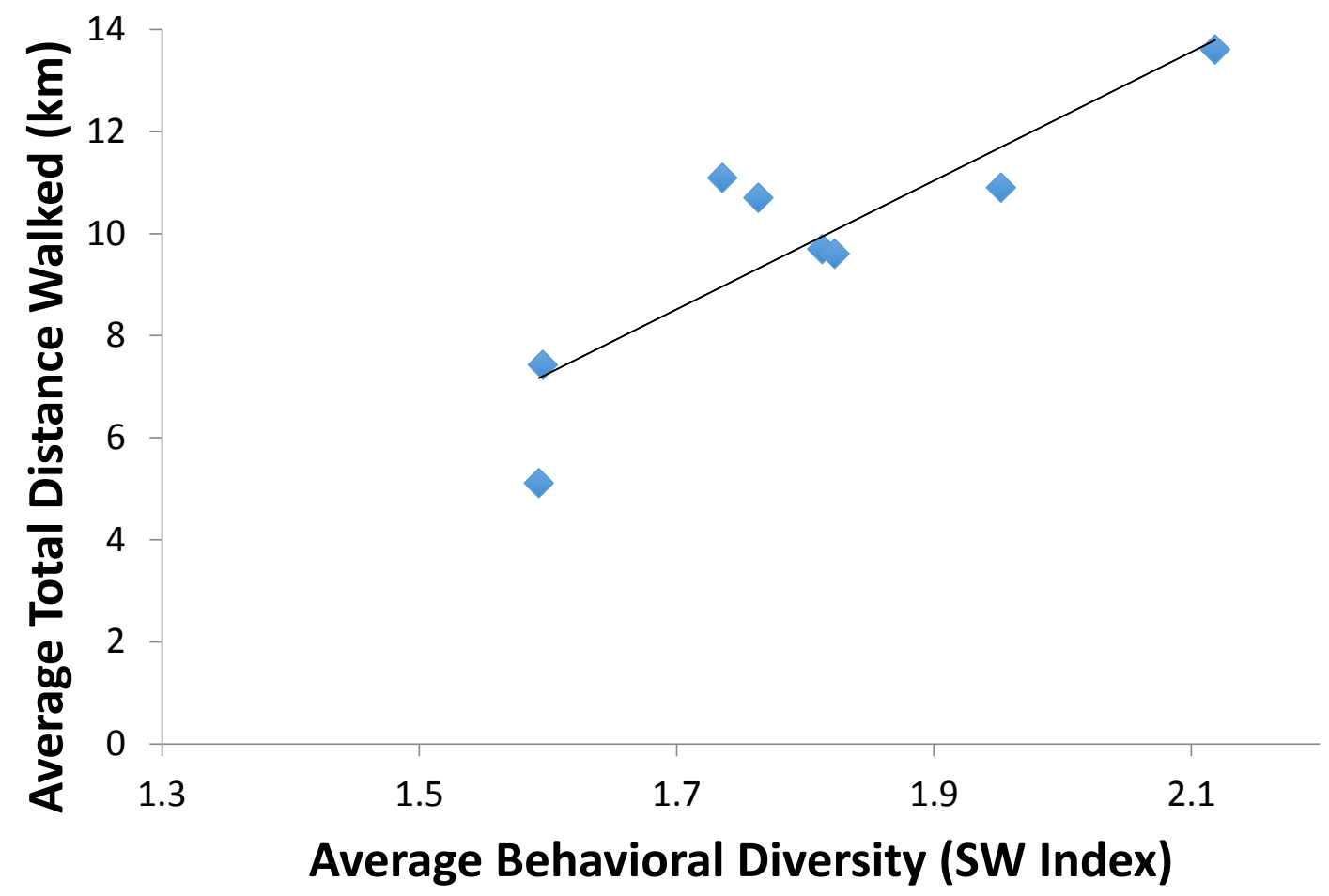

Fig. 9 The average behavioral diversity and average total distance walked for the $22 \mathrm{hr}$ treatment period. This relationship was strong related for treatment Both $(\mathrm{R}=0.86)$. 


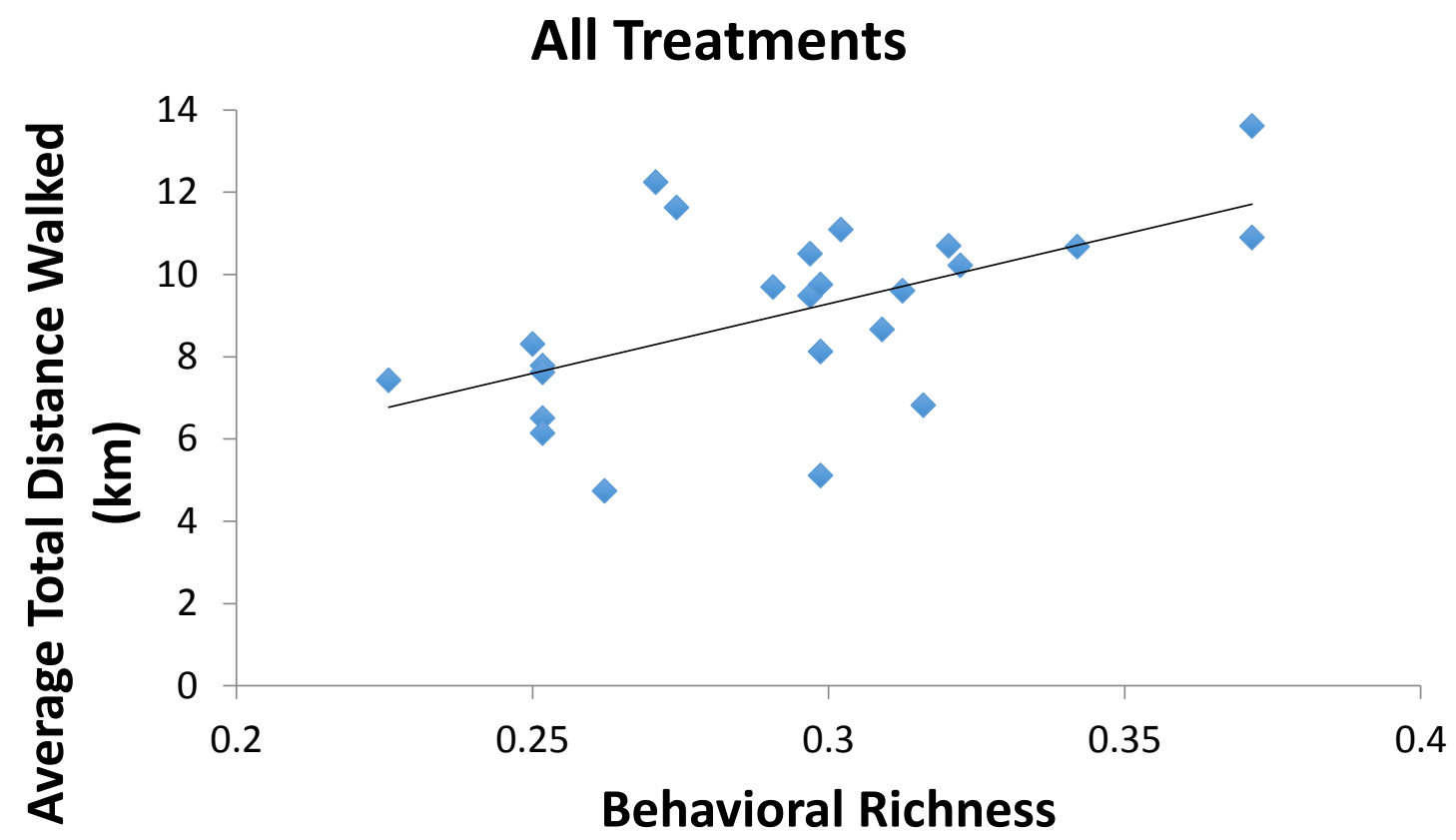

Fig. 10 The average behavioral richness and average total distance walked for the $22 \mathrm{hr}$ treatment period. This relationship was moderate when all treatments were considered $(\mathrm{R}$ $=0.57)$. 


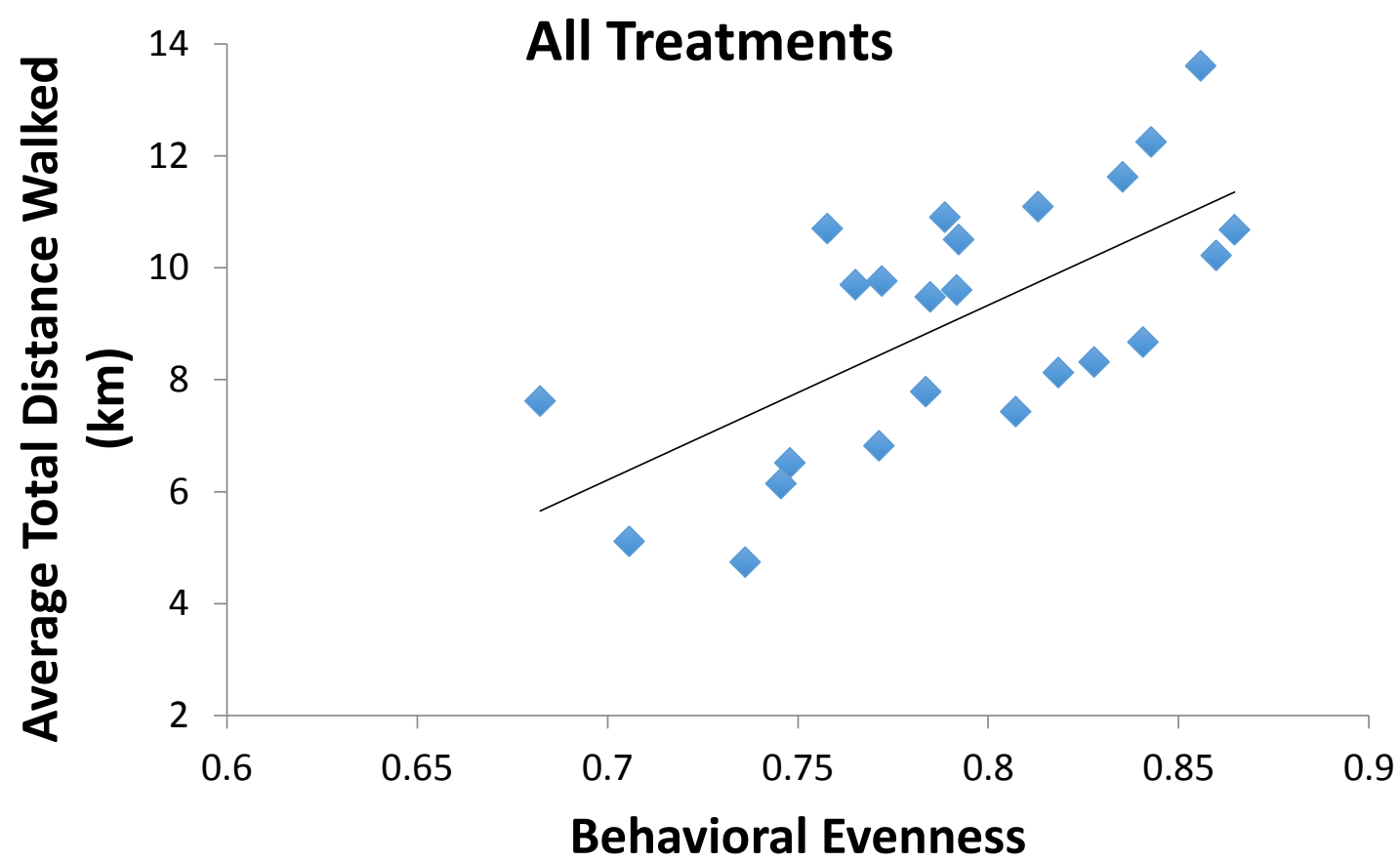

Fig. 11 The average behavioral evenness and average total distance walked for the $22 \mathrm{hr}$ treatment period. This relationship was moderate when all treatments were considered ( $\mathrm{R}$ $=0.66)$. 


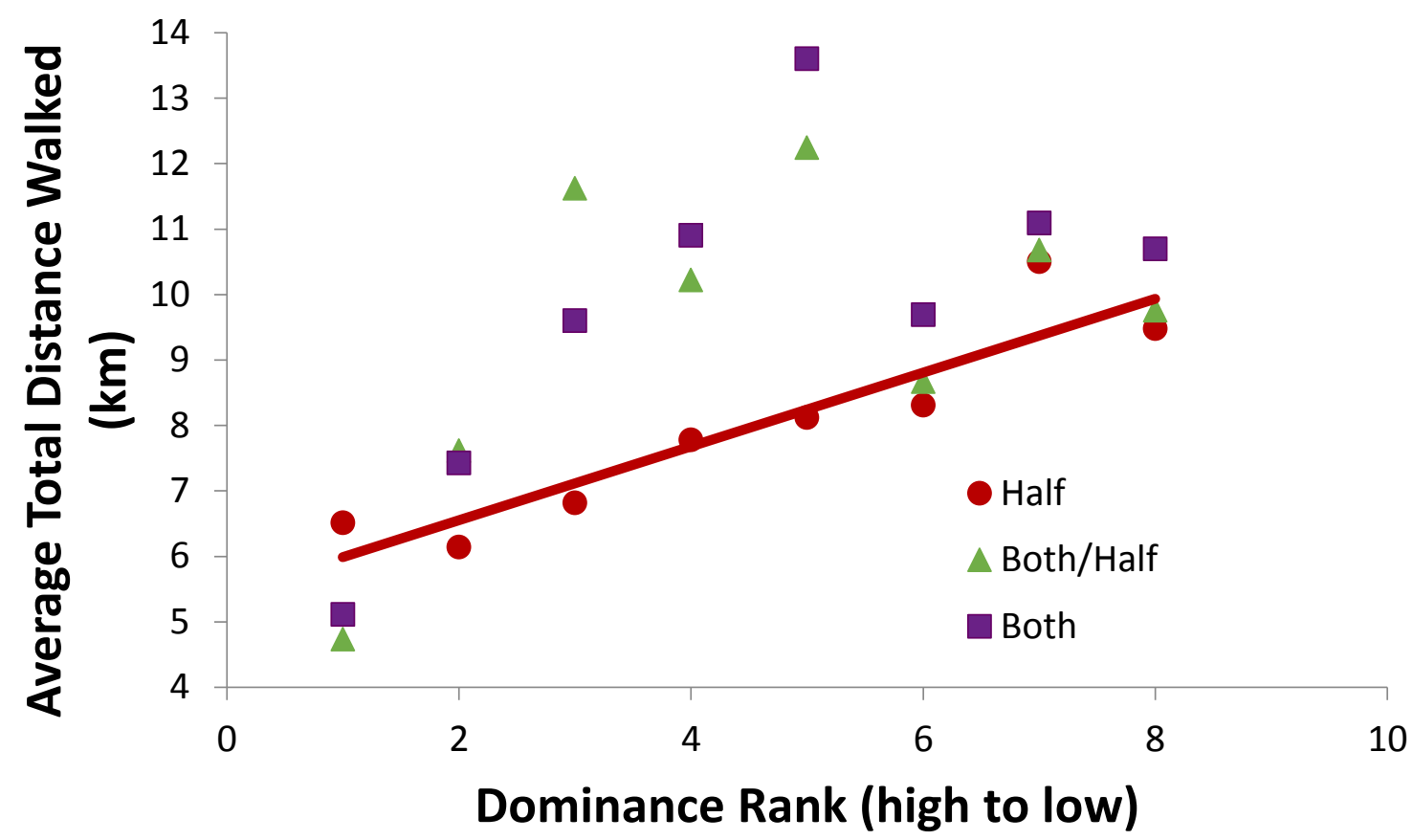

Fig. 12 The total average distance walked relative to the rank of the elephant from most dominant to least dominant for each of the three treatments. Dominance rank and total distance were found to be significantly correlated for treatment Half $\left(\mathrm{P}=0.001, \mathrm{R}^{2}=\right.$ $0.85)$, but not related for treatment Both/Half $\left(\mathrm{P}=0.18, \mathrm{R}^{2}=0.28\right)$ or treatment Both $(\mathrm{P}=$ $\left.0.06, \mathrm{R}^{2}=0.47\right)$ 
A

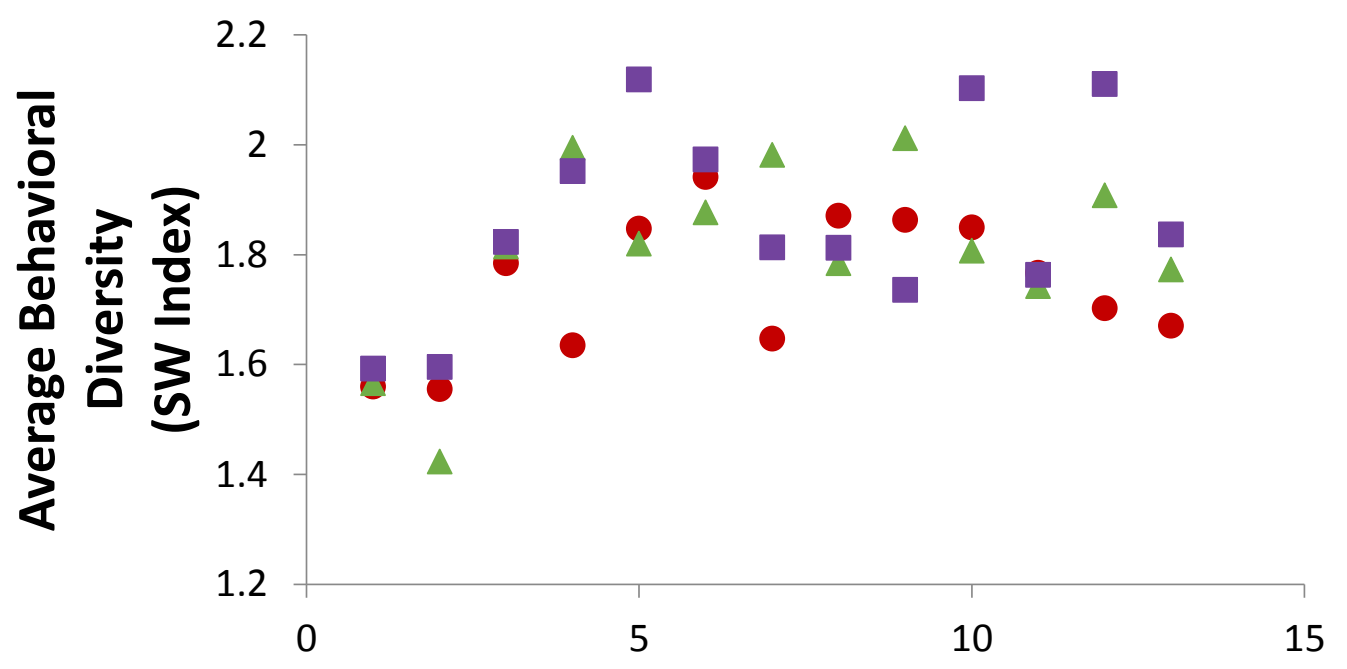

B

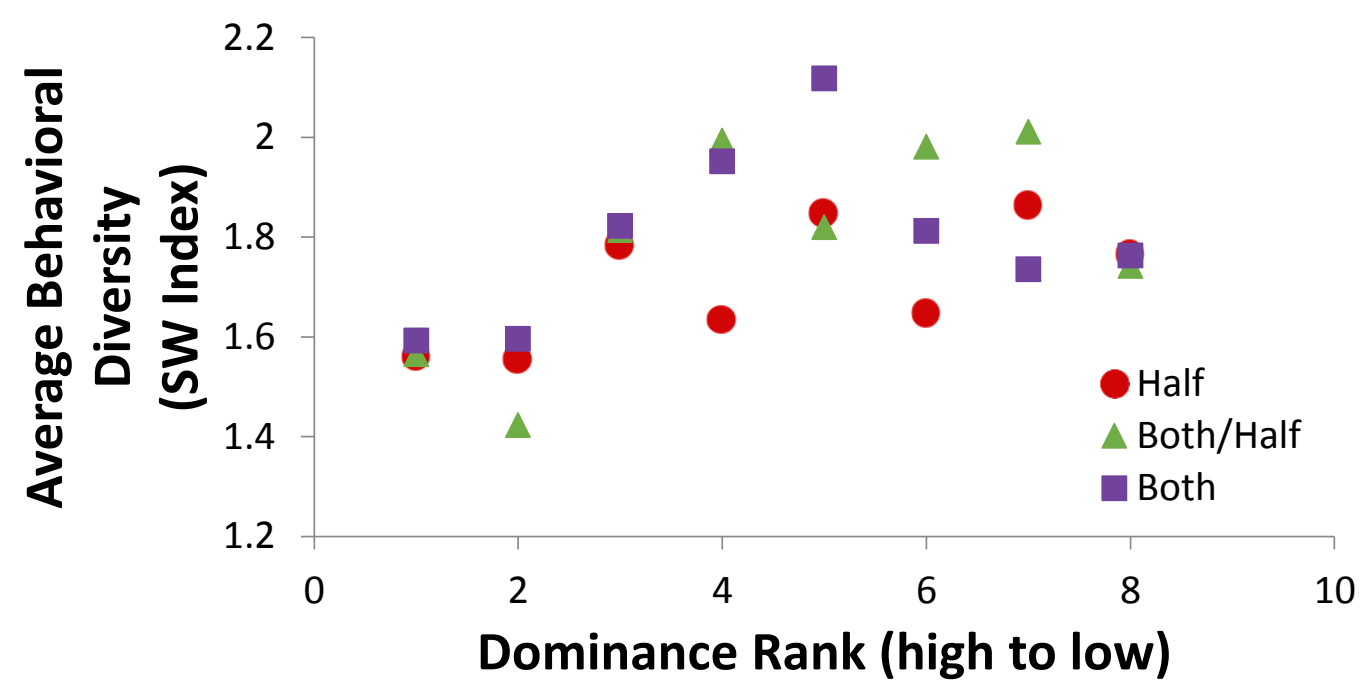

Fig. 13A and 13B A) The average behavioral diversity relative to the rank of the elephant from most dominant to least dominant for each of the three treatments for all 13 elephants. No significant relationship was found for treatments Half $\left(\mathrm{P}=0.27, \mathrm{R}^{2}=\right.$ 0.11), Both/Half $\left(P=0.18, R^{2}=0.16\right)$, or Both $\left(P=0.18, R^{2}=0.16\right)$. B) The average behavioral diversity relative to the rank of the elephant from most dominant to least dominant for each of the three treatments for those elephants equipped with GPS anklets. No significant relationship was found for treatments Half $\left(P=0.66, R^{2}=0.08\right)$, Both/Half $\left(\mathrm{P}=0.10, \mathrm{R}^{2}=0.62\right)$, or Both $\left(\mathrm{P}=0.41, \mathrm{R}^{2}=0.34\right)$. 
A

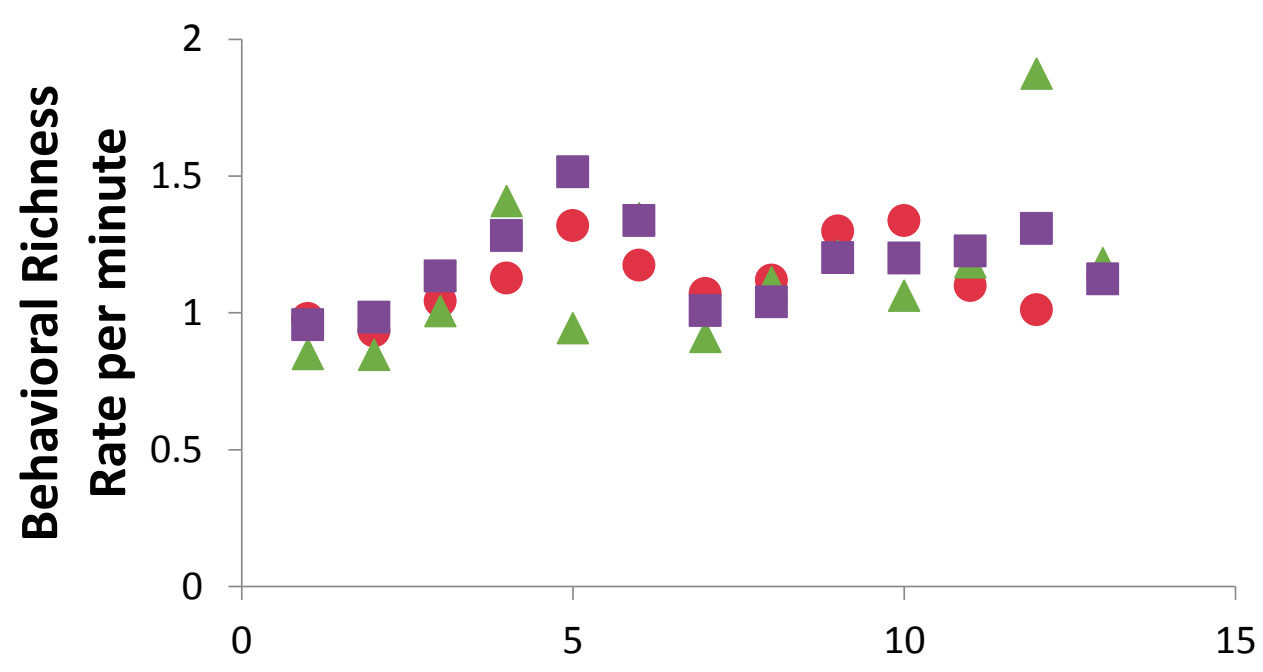

B

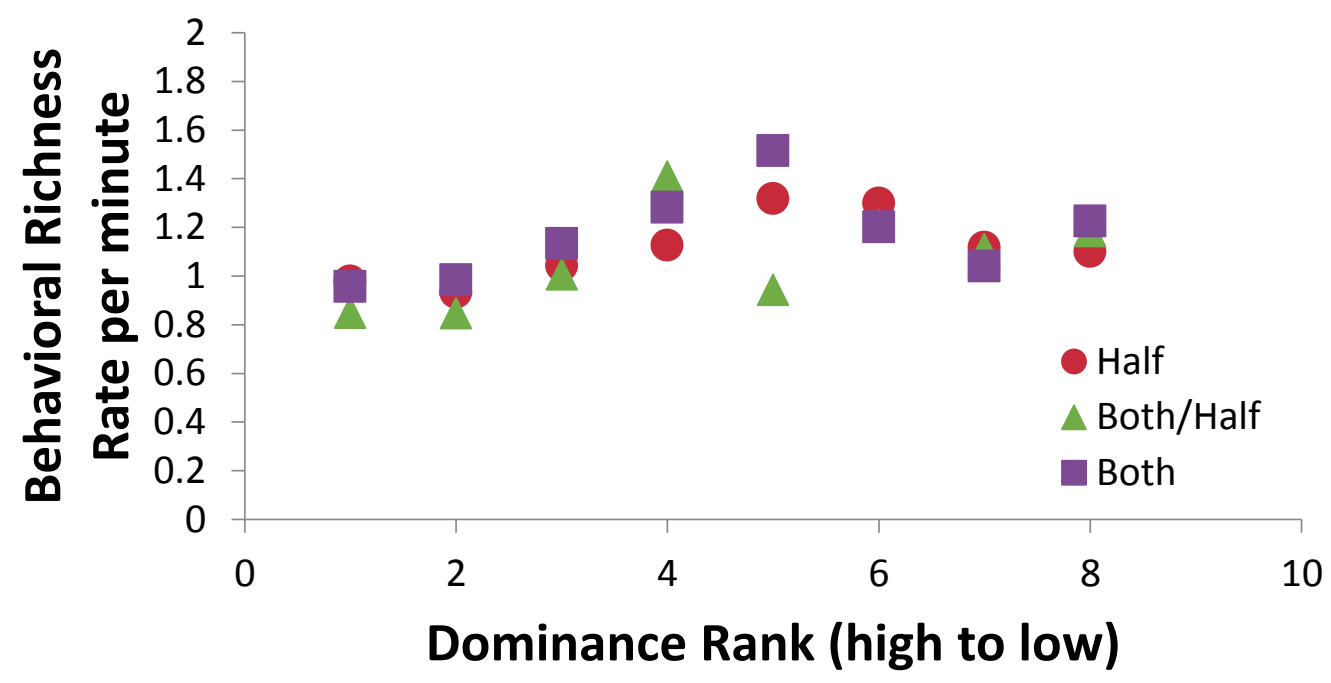

Fig. 14A and 14B A) The average behavioral richness rate per minute relative to the rank of the elephant from most dominant to least dominant for each of the three treatments for all 13 elephants. No significant relationship was found for treatments Half $\left(P=0.26, R^{2}=\right.$ 0.11), Both/Half $\left(P=0.07, R^{2}=0.27\right)$, or Both $\left(P=0.47, R^{2}=0.05\right)$. B) The average behavioral richness relative to the rank of the elephant from most dominant to least dominant for each of the three treatments for those elephants equipped with GPS anklets. No significant relationship was found for treatments Half $\left(P=0.13, R^{2}=0.34\right)$, Both/Half $\left(\mathrm{P}=0.14, \mathrm{R}^{2}=0.32\right)$, or Both $\left(\mathrm{P}=0.31, \mathrm{R}^{2}=0.17\right)$. 

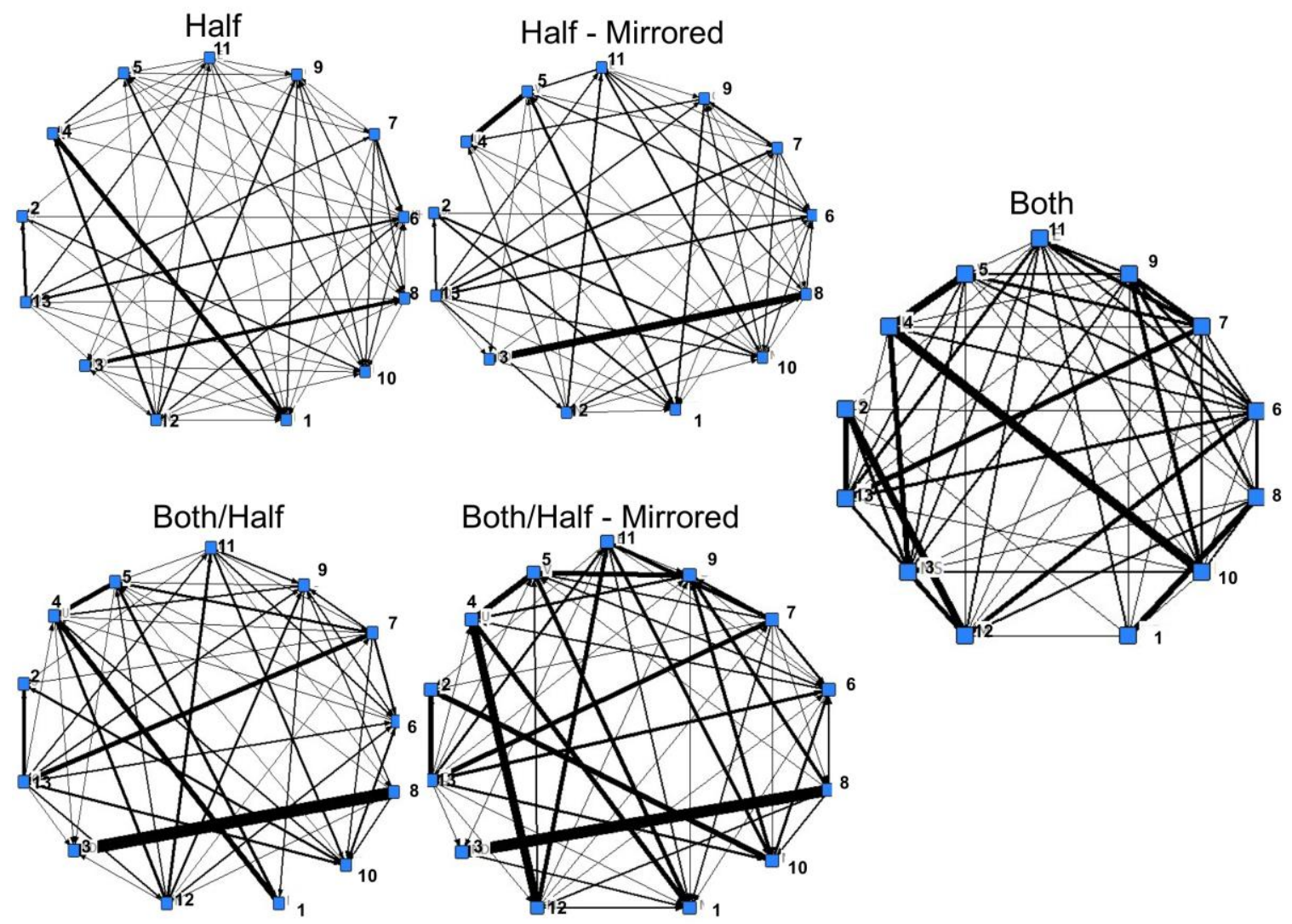

Fig. 15 The level of association between any two elephants based upon numerical values from the simple index calculations across treatments of space and food. Level of association between the pair is indicated by arrow boldness. The bolder the arrow, the greater the level of association. Numbers refer to individuals listed in Table 2. 


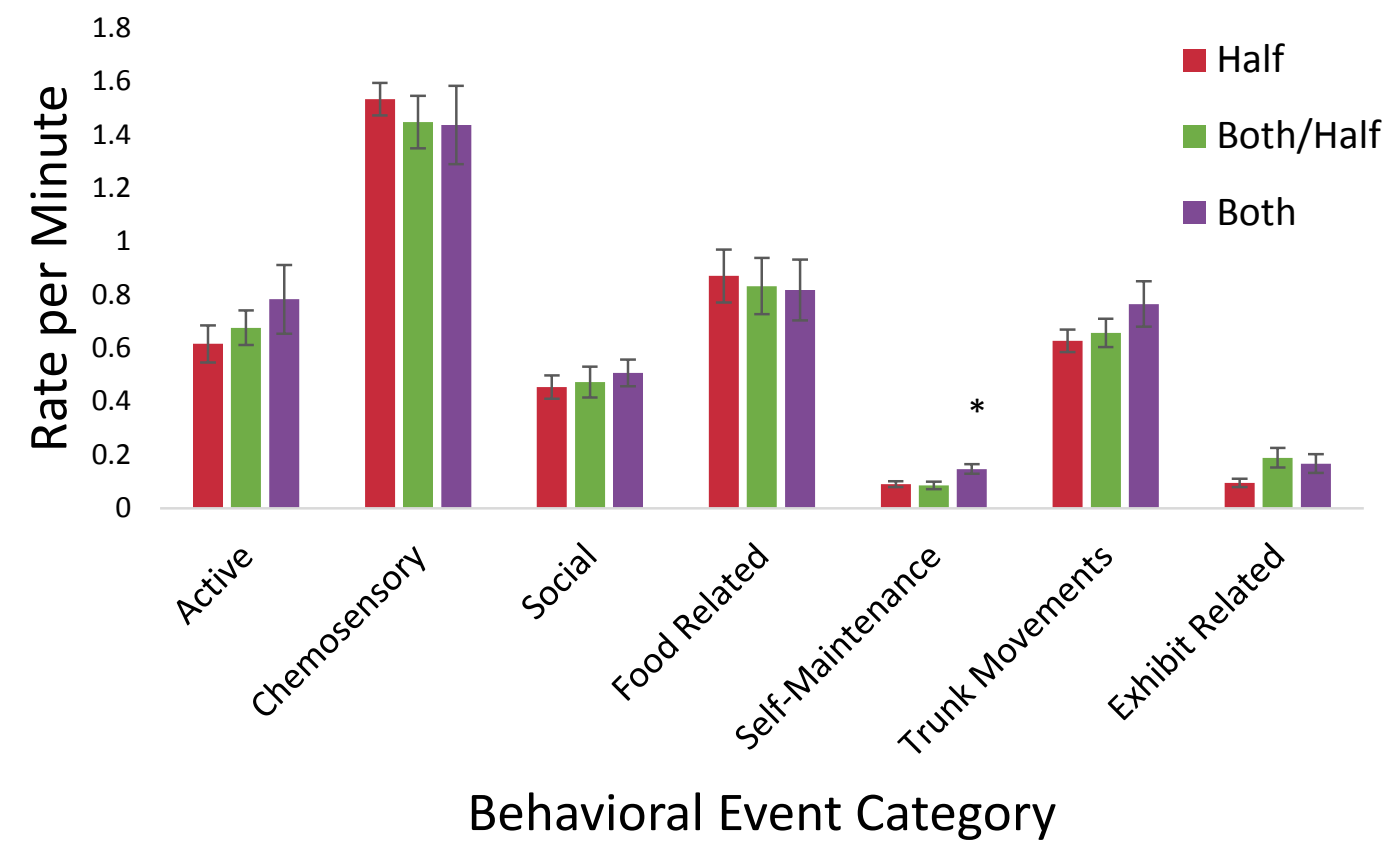

Fig. 16 The rates per minute of behavioral events collected via all occurrence sampling across the three treatments of space and food. A significant difference in selfmaintenance behavior (urination and defecation) between Both and Half $(\mathrm{P}=0.019)$ as well as Both/Half $(\mathrm{P}=0.023)$ was determined. 


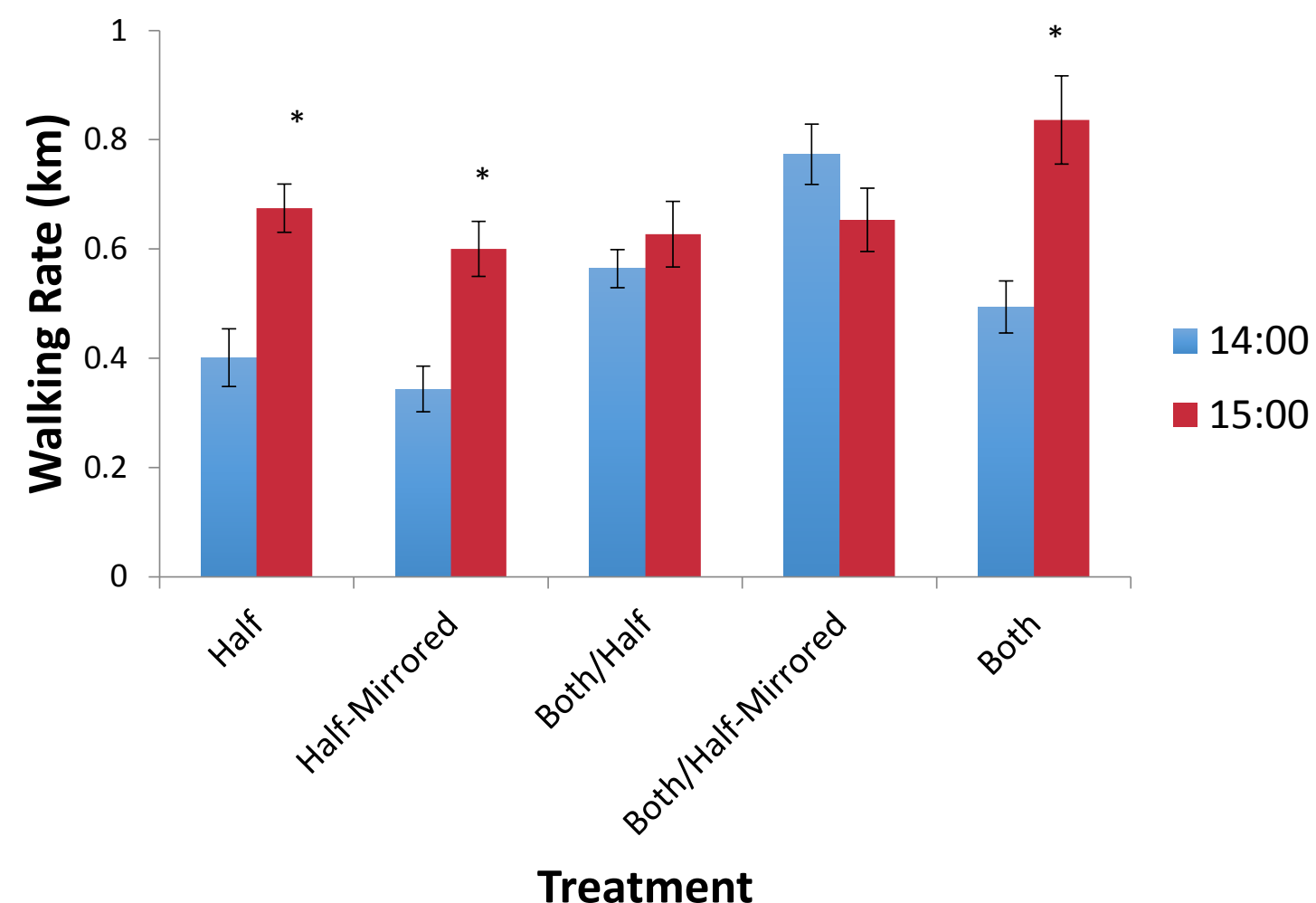

Fig. 17 The average distance walked one hour before (2:00 PM) and one hour after (3:00 PM) produce and pellets were tossed into the exhibit space. Significant increases in walking activity $1 \mathrm{hr}$ before and $1 \mathrm{hr}$ after tossed produce and pellets were found for treatments Half (test statistic value $=2.55, \mathrm{P}=0.003$ ), Half-Mirrored (test statistic value $=$ 3.12, $\mathrm{P}=0.03$ ) and Both (test statistic value $=7.67, \mathrm{P}=0.0003$ ). Bars are $\pm 1 \mathrm{SEM}$. 


\section{LITERATURE CITED}

Altman JD. 1998. Animal activity and visitor learning at the zoo. Anthrozoo 11:12-21.

Altmann J. 1974. Observational study of behavior: sampling methods. Behaviour 49:227266.

Anderson C, Arun AS, Jensen P. 2010. Habituation to environmental enrichment in captive sloth bears: Effect on stereotypies. Zoo Biol 29:705-714.

Andrews J, Mecklenborg M, Bercovitch FB. 2004. Milk intake and development in a newborn captive African elephant (Loxodonta africana). Zoo Bio 24:275-281.

Archie EA, Morrison TA, Foley CAH, Moss CJ, Alberts SC. 2006a. Dominance rank relationships among wild female African elephants, Loxodonta africana. Anim Behav 71:117-127.

Archie EA, Moss CJ, Alberts SC. 2006b. The ties that bind: genetic relatedness predicts the fission and fusion of social groups in wild African elephants. Proc R Soc B 273:513-522.

Audubon Nature Institute. 2013. New elephant exhibit and lazy river coming to zoo. http://www.auduboninstitute.org/media/releases/new-elephant-exhibit-and-lazyriver-coming-zoo. Retrieved November 22, 2013.

Bates LA, Poole JK, Byrne RW. 2008. Elephant cognition. Curr Biol 18:544-546.

Beardsworth A, Bryman A. 2001. The wild animal in late modernity: the case of the Disneyization of zoos. Tour Stud 1:83-104.

Belz EE, Kennell JS, Czambel RK, Rubin RT, Rhodes ME. 2003. Environmental enrichment lowers stress-responsive hormones in singly housed male and female rats. Pharmacol Biochem and Behav 76:481-486.

Björk K. 2011. The effect of extra feed supply on stereotypic behaviour in Asian elephants (Elephas maximus) (thesis). Linköping, Sweden: Linköping University.

Bryant MJ, Ewbank R. 1972. Some effects of stocking rate and group size upon agonistic behaviour in groups of growing pigs. Br Vet J 128:64-50.

Buckley C. 2008. Captive elephant foot care: natural-habitat husbandry techniques. In: Csuti B, Sargent E, Bechert U, editors. The elephant's foot: prevention and care of foot conditions in captive Asian and African elephants. Ames: Iowa State University Press. p. 53-55.

Carlstead K, Seldensticker J, Baldwin R. 1991. Environmental enrichment for zoo bears. Zoo Biol 10: 3-16.

Cheyenne Mountain Zoological Society. 2013. Encounter Africa. http://www.cmzoo.org/exhibitsAttractions/encounterAfrica/. Retrieved November 22, 2013.

Cipreste CF, de Azevedo CS, Young RJ. 2010. How to develop a zoo based environmental enrichment program: incorporating environmental enrichment into exhibits. In: Kleiman D, Thompson K, Baer C, editors. Wild mammals in captivity. Chicago: University of Chicago Press. p. 171-180. 
Clark FE, Melfi VA. 2011. Environmental enrichment for a mixed-species nocturnal mammal exhibit. Zoo Bio 29:1-17.

Cleveland Metroparks. 2013. Zoo History. http://www.clemetzoo.com/Zoo/ZooHistory.aspx. Retrieved November 22, 2013.

Clubb R, Mason G. 2003a. A review of the welfare of zoo elephants in Europe. RSPCA. Oxford: University of Oxford, Animal Behavior Research Group.

Clubb R, Mason G. 2003b. Captivity effects on wide-ranging carnivores. Nature 425:473-474.

Clubb R, Rowcliffe M, Lee P, Mar KU, Moss C, Mason GJ. 2008. Compromised survivorship, fecundity and population persistence in zoo elephants. Science 322:1649-1649.

Cohn JP. 1992. Decisions at the zoo. BioScience 42:654-659.

Colbert D. 2010. Enrichment. Association of Zoos and Aquariums. www.aza.org/enrichment/. Retrieved February 11, 2013.

Dallas Zoo. 2013. Elephants on the savanna http://www.dallaszoo.com/exhibitsexperiences/giants-of-the-savanna/elephants-on-the-savanna/. Retrieved November 22, 2013.

Dallaire JA, Meagher RK, Mason GJ. 2011. Individual differences in stereotypic behaviour predict individual differences in the nature and degree of enrichment use in caged American mink. Appl Anim Behav Sci 142:98-108.

Denver Zoo. 2013. Toyota Elephant Passage. http://www.denverzoo.org/toyota elephant_passage/plan_yourvisit/exhibit/index.html. Retrieved November 22, 2013.

Desmond TJ, Laule G. 1991. Protect-contact elephant training. AAZAPA Annual Conference Proceedings. Silver Spings, MD. P. 606-613.

Douglas-Hamilton I, Bhalla S, Wittemyer G, Vollrath F. 2006. Behavioural reactions of elephants towards a dying and deceased matriarch. App Anim Behav Sci 100:87102.

Elzanowski A, Sergiel A. 2006. Stereotypic behavior of a female Asiatic elephant (Elephas maximus) in a zoo. J Appl Anim Welf Sci 9:223-232.

Escondido - City of Choice. 2013. Escondido, California Weather. http://www.escondido.org/weather-news.aspx. Retrieved April 10, 2013

Evans KE, Harris S. 2008. Adolescence in male African elephants, Loxodonta africana, and the importance of sociality. Anim Behav 76:779-787.

Foerder P, Galloway M, Barthel T, Moore DE, Reiss D. 2011. Insightful problem solving in an Asian elephant. PLoS ONE 6:e23251.

Foley C, Papageorge S, Wasser S. 2001. Noninvasive stress and reproductive measures of social and ecological pressures in free-ranging African elephants. Conserv Biol 15:1134-1142. 
Freeman EW, Schulte BA, Brown JL. 2010. Using behavioral observations and keeper questionnaires to assess social relationships among captive female African elephants. Zoo Biol 29:140-153.

Gadgil M, Nair PV. 1984. Observation on the social behaviour of free ranging groups of tame Asiatic elephant (Elephas maximus Linn). Proc Indian Acad Sci 93:225-233.

Ginsberg JR, Young TP. 1992. Measuring assocation between individuals or groups in behavioural studies. Anim Behav 44:377-379.

Goodyear S. 2015. Habituation to auditory stimuli by captive African elephants (Loxodonta africana) (thesis). Bowling Green, KY: Western Kentucky University.

Greco BJ, Brown TK, Andrews JRM, Swaisgood RR, Caine NG. 2013. Social learning in captive African elephants. Anim Cogn 16:459-469.

Hacker CE, Horback KM, Miller LJ. 2015. GPS technology as a proxy tool for determining relationships in social animals: An example with African elephants. Appl Anim Behav Sci 163:175-182.

Hediger H. 1955. Studies of the psychology and behavior of captive animals in zoos and circuses. New York, New York: Criterion Press. 166 p.

Holdgate MR. 2015. Applying GPS and accelerometers to the study of African savanna (Loxodonta africana) and Asian elephant (Elephas maximus) welfare in zoos (dissertation). Portland, OR: Portland State University.

Horback K, Miller L, Andrew J, Kuczaj S, Anderson M. 2012. The effects of GPS collars on African elephant (Loxodonta africana) behavior at the San Diego Zoo Safari Park. Appl Anim Behav Sci 142:76-81.

Horton GMJ, Malinowski K, Burgher CC, Palatini DD. 1991. The effect of space allowance and sex on blood catecholamines and cortisol, feed consumption and average daily gain in growing lambs. App Anim Behav Sci 32:197-204.

Hosey GR. 2005. How does the zoo environment affect the behaviour of captive primates? Appl Anim Behav Sci 90:107-129.

Hosey G. 2008. Review: A preliminary model of human-animal relationships in the zoo. App Anim Behav Sci 109:105-127.

Hutchins M. 2006. Variation in nature: Its implications for zoo elephant management. Zoo Biol 25:161 -171.

Johansson E. 2009. Food preference in African Elephants (Loxodonta africana) and the impact of bomas in the vicinity of and in Masai Mara National Reserve. First cycle, G2E. Skara: SLU, Dept. of Animal Environment and Health.

Koene P. 1995. The use of time budget studies in captive propagation and zoo biology. In: Gansloßer, Hodges JK, Kaumanns W, editors. Research and captive propagation. Fürth, Germany: Filander Verlag. p 271-284.

Koene P. 2013. Behavioral ecology of captive species: using behavioral adaptations to assess and enhance welfare of nonhuman zoo animals. J Appl Anim Welf Sci 16:360-380. 
Laule G. 2003. Positive reinforcement training and environmental enrichment: enhancing animal well-being. J Amer Vet Med Assoc 223:969-973.

Lee, PC. 1987. Allomothering among African elephants. Anim Behav 35:278-291.

Leighty K, Soltis J, Savage A. 2010. GPS assessment of the use of exhibit space and resources by African elephants (Loxodonta africana). Zoo Biol 29:220-220.

Levy DM. 1944. On the problem of movement restraint: tics, stereotyped movements, hyperactivity. Am J Orthopsychiat 14:644-671.

Lewis J. 2010. Diary Entries. Elephants of Asia at the L.A. Zoo. http://www.lazoo.org/animals/elephantsofasia/diary/. Retrieved November 22, 2013.

Little KA, Sommer V. 2002. Change of enclosure in Langur monkeys: implications for the evaluation of environmental enrichment. Zoo Bio 21:549-559.

Loizi H, Goodwin TE, Rasmussen LEL, Whitehouse AM, Schulte BA. 2009. Sexual dimorphism in the performance of chemosensoy investigatory behaviours by African elephants (Loxodonta africana). Behaviour 146:373-392.

Maple TL. 2014. Elevating the priority of zoo animal welfare: the chief executive as an agent of reform. Zoo Bio 33:1-7.

Marchant-Forde JN. 2015. The science of animal behavior and welfare: challenges, opportunities and global perspective. Front in Vet Sci 2:16.

Margulis SW, Hoyos C, Anderson M. 2003. Effect of felid activity on zoo visitor interest. Zoo Bio 22:587-599.

Martin P, Bateson P. 2007. Measuring behaviour: an introductory guide. 3rd Edition. New York, NY: Cambridge University Press. 187 p.

Mason, GJ. 1991. Stereotypies: a crtical review. Anim Behav 41:1015-1037.

McComb K, Moss C, Durant S, Baker L, Sayialel S. 2001. Matriarchs as repositories of social knowledge in African elephants. Sci Mag 292:491-494.

Mellen JD, Stevens VJ, Markowitz H. 1981. Environmental enrichment for servals, Indian elephants and Canadian otters at Washington Park Zoo, Portland. Int Zoo Yb 21:196-201.

Miller L. 2012. Visitor reaction to pacing behavior: influence on the perception of animal care and interest in supporting zoological institutions. Zoo Bio 31:242-248.

Miller L, Chase MJ, Hacker CE. (in press). A comparison of walking rates between wild and zoo African elephants. J Appl Anim Welf Sci.

Miller L, Andrews J, Anderson M. 2011. Validating methods to determining walking rates of elephants within a zoological institution. Anim Welf 22:577-582.

Moreira N, Brown JL, Moraes W, Swanson WF, Monteiro-Filho ELA. 2007. Effect of housing and environmental enrichment on adrenocortical activity, behavior and reproductive cyclicity in the female tigrina (Leopardus tigrinus) and margay (Leopardus wiedii). Zoo Bio 26:441-460. 
Murphy ES, McSweeney FK, Smith RG, McComas JJ. 2003. Dynamic changes in reinforce effectiveness: theoretical, methodological and practical implications for applied research. J App Behav Ana 36:421-438.

Murray CM, Sandeep VM, Pusey AE. 2007. Dominance rank influences female space use in wild chimpanzees, Pan troglodytes: towards an ideal despotic distribution. Anim Behav 74:1795-1804.

O’Connor T, Goodman P, Clegg B. 2007. A functional hypothesis of the threat of local extirpation of woody plant species by elephant in Africa. Biol Conserv 136:329345.

Olson D. 2004. Elephant husbandry resource guide. Lawrence: Allen Press. 280 p. Oregon Zoo.

Pearce GP, Patterson AM. 1993. The effect of space restriction and provision of toys during rearing on the behavior, productivity, and physiology of male pigs. Appl Anim Behav Sci 36:11-28.

Pepper JW, Mitani JC, Watts DP. 1999. General gregariousness and specific social preferences among wild Chimpanzees. Int J Primatol 20:613-632.

Plotnik JM, Lair R, Suphackoksahakun W, de Waal FBM. 2011. Elephants know when they need a helping trunk in a cooperative task. Proc Natl Acad Sci USA 108:5116-5121.

Poole J. 1996. The African elephant. In: Kangwana K, editor. Studying elephants. Nairobi: Kenya. The African Wildlife Foundation. p 1-9.

Poole J, Granuli, P. 2009. Mind and movement: meeting the interests of elephants. In: Forthman DL, Kane FL, Hancocks D, Waldau, PF, editors. An elephants in the room: the science and wellbeing of elephants in captivity. North Grafton: Tufts Center for Animals \& Public Policy. p 1-21.

Posta B. 2011. The effects of housing and enrichment on zoo elephant behavior. (thesis). Bowling Green, OH: Bowling Green State University.

Posta B, Huber R, Moore DE. 2013. The effects of housing on zoo elephant behavior: a quantitative case study of diurnal and season variation. Int J Comp Psych 26:3752.

Quirke T, O'Riordan RM. 2011. The effect of a randomized enrichment treatment schedule on the behaviour of cheetahs (Acinonyx jubatus). App Anim Behav Sci 135:103-109.

Rees PS. 1982. Gross assimilation efficiency and food passage time in the African elephants. Afr J Eco 20:193-198.

Rees PS. 2009. Activity budgets and the relationship between feeding and stereotypic behaviors in Asian elephants (Elephas maximus) in a zoo. Zoo Bio 28:79-97.

Renner MJ, Lussier JP. 2002. Environmental enrichment for the captive spectacled bear (Tremarctos ornatus). Pharm, Biochem \& Behav 73:279-284. 
RSPCA Australia Scientific Seminar. 2007. How much space does an elephant need? The impact of confinement on animal welfare. J Vet Behav 2:185-187.

“San Diego Zoo Safari Park Elephant Valley.” 3305’58.16” N and 11659'51.59 W. Google Earth. April 4, 2015. May 6, 2015.

Schulte B. 2000. Social structure and helping behavior in captive elephants. Zoo Biol 5:447-459.

Schwartz R. 2009. Elephant odyssey opens in two months. San Diego Zoo. http://blogs.sandiegozoo.org/2009/03/24/elephant-odyssey-opens-in-two-months/. Retrieved November 22, 2013.

Senft RL, Coughenour MB, Rittenhouse LR, Sala OE, Swift DM. 1987. Large herbivore foraging and ecological hierarchies. BioScience 37:11.

Shannon G, Page B, Duffy K, Slotow R. 2006. The role of foraging behaviour in the sexual segregation of the African elephant. Oecologia 150:344-354.

Shannon CE, Weaver W. 1949. The mathematical theory of communication. Champaign: University of Illinois Press. P 117.

Shepherdson DJ, Carlstead K, Mellen JD, Seidensticker J. 1993. The influence of food presentation of the behavior of small cats in confined environments. Zoo Bio 12:203-216.

Shepherdson DJ. 2010. Principles of and research on environmental enrichment for mammals. In: Kleiman D, Thompson K, Baer C, editors. Wild mammals in captivity. Chicago: University of Chicago Press. p 62-67.

Stoinski TS, Daniel E, Maple TL. 2000. A preliminary study of the behavioral effects of feeding enrichment on African elephants. Zoo Bio 6:485-493.

Stokke S, Du Toit JT. 2002. Sexual segregation in habitat use by elephants in Chobe National Park, Botswana. Afr J Eco 40:360-371.

Swaisgood RR, Shepherdson DJ. 2005. Scientific approaches to enrichment and stereotypies in zoo animals: what's been done and where should we go next? Zoo Bio 24:499-518.

Tarou L, Bashaw M. 2007. Maximizing the effectiveness of environmental enrichment: suggestions from the experimental analysis of behavior. Appl Anim Behav Sci 102:189-204.

Theiss A, Yen DC, Ku C. 2005. Global Positioning Systems: an analysis of applications, current development, and future implementations. Comput Stand and Interfaces 27:89-100.

Tresz H. 2006. Behavioral management at the Phoenix Zoo: new strategies and perspectives. J of Appl Anim Welf Sci 91:65-70.

Veasey JS. 2006. Concepts in the care and welfare of captive elephants. Int Zoo Yb 40:63-79.

Weather Underground. 2015. Weather History for KRNM. http://www.wunderground. 
com/history/airport/KRNM/2014/7/30/MonthlyHistory.html?req_city=Escondido \&req_state $=$ CA\&req_statename $=\&$ reqdb.zip $=92025 \&$ reqdb.magic $=1 \&$ reqdb.wm o=99999. Retrieved August 29, 2015.

Wells D. 2009. Sensory stimulation as environmental enrichment for captive animals: a review. Appl Anim Behav Sci 118:1-11.

Wittemyer G, Douglas-Hamilton I, Getz WM. 2005. The socioecology of elephants: analysis of the processes creating multitiered social structure. Anim Behav 69:1357-1371.

Wittemyer G, Getz WM. 2007. Hierarchical dominance structure and spatial organization in African elephants, Loxodonta africana. Anim Behav 73:671-681

Wittemyer G, Getz WM, Vollrath F, Douglas-Hamilton I. 2007. Social dominance, seasonal movements, and spatial segregation in African elephants: a contribution to conservation behavior. Behav Ecol Sociobiol 61:1919-1931. 